NIST Special Publication 1200-21

\title{
Characterization of nanoparticle suspensions using single particle inductively coupled plasma mass spectrometry
}

Version 1.0

\author{
K. E. Murphy \\ J. Liu \\ A. R. Montoro Bustos \\ M. E. Johnson \\ M. R. Winchester
}

This publication is available free of charge from:

http://dx.doi.org/10.6028/NIST.SP.1200-21 


\section{Characterization of nanoparticle suspensions using single particle inductively coupled plasma mass spectrometry}

Version 1.0

K. E. Murphy

A. R. Montoro Bustos

M. E. Johnson

M. R. Winchester Chemical Sciences Division Material Measurement Laboratory

J. Liu Material Measurement Science Division Material Measurement Laboratory

This publication is available free of charge from:

http://dx.doi.org/10.6028/NIST.SP.1200-21

December 2015

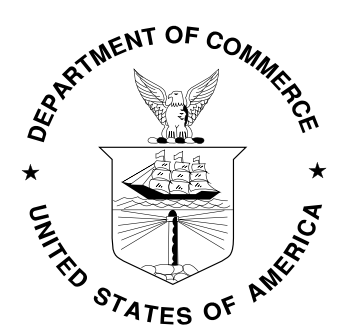

U.S. Department of Commerce Penny Pritzker, Secretary

National Institute of Standards and Technology Willie May, Under Secretary of Commerce for Standards and Technology and Director 
Certain commercial entities, equipment, or materials may be identified in this document in order to describe an experimental procedure or concept adequately. Such identification is not intended to imply recommendation or endorsement by the National Institute of Standards and Technology, nor is it intended to imply that the entities, materials, or equipment are necessarily the best available for the purpose.

National Institute of Standards and Technology Special Publication 1200-21

Natl. Inst. Stand. Technol. Spec. Publ. 1200-21, 29 pages (December 2015)

CODEN: NSPUE2

This publication is available free of charge from: http://dx.doi.org/10.6028/NIST.SP.1200-21 


\section{FOREWORD}

This National Institute of Standards and Technology (NIST) special publication (SP) is one in a series of NIST SPs that address research needs articulated in the National Nanotechnology Initiative (NNI) Environmental, Health, and Safety Research Strategy published in 2011 [1]. This Strategy identified a Nanomaterial Measurement Infrastructure (NMI) as essential for science-based risk assessment and risk management of nanotechnology-enabled products as pertaining to human health, exposure, and the environment. NIST was identified as the lead federal agency in the NMI core research area of the Strategy; this research area includes measurement tools for the detection and characterization of nanotechnology-enabled products. Single particle inductively coupled plasma mass spectrometry (spICP-MS) is emerging as a promising analytical method for the characterization of nanoparticles (NPs) in natural matrices at environmentally relevant concentrations. The rapid development of spICP-MS for counting and sizing of NPs has resulted in a wide range of recommended metrological conditions for use in the implementation of this method.

The objective of this SP is to establish a protocol for the determination of mean nanoparticle size (equivalent spherical particle diameter), number based size distribution, particle number concentration and mass concentration of ions in an aqueous suspension of NPs using spICP-MS. The example presented in this SP pertains to the measurement of gold nanoparticles (AuNPs) and silver nanoparticles (AgNPs), but the presented protocol is applicable to the measurement of all spherical nanoparticles containing elements measureable by ICP-MS. In addition, this protocol describes a Kragten spreadsheet approach for estimation of the expanded uncertainty of the spICP-MS particle size and particle number concentration measurement.

As the method advances, improvements will be realized and updates to this protocol may be released in the future. Visit http://www.nist.gov/mml/nanoehs-protocols.cfm to check for revisions and additions to this protocol or for new protocols in the series. We also encourage users to report citations to published work in which this protocol has been applied. 


\section{Introduction}

Fundamental to the study of environmental and human health effects of engineered nanomaterials (ENMs) is the ability to characterize the material properties of the ENMs used in the studies within a dose range and in media commensurate with realistic exposure scenarios. Many of the traditional methods for characterizing nanoparticles (NPs) do not have the capability to perform the "in situ" measurements required to establish an accurate assessment of the potential hazards associated with ENMs. Single particle detection using inductively coupled plasma mass spectrometry (spICP-MS) is a sensitive and selective method capable of direct analysis of individual NPs in suspension and which can simultaneously provide information on size, size distribution, particle number concentration, aggregation state, and ionic content.

\section{Principles and Scope}

The theoretical basis for spICP-MS was first outlined by Degueldre et al. [2]. Measurements are performed on commercially available ICP-MS instruments and are acquired in time resolved mode using short (micro second to millisecond), consecutive, measurement periods referred to as dwell times $\left(t_{\text {dwell }}\right)$. spICP-MS relies on the principle that as a NP suspended in a solution is atomized and ionized in the plasma, it will produce a spatially concentrated packet of ions which is measured as a transient signal spike superimposed on the steady-state signal produced by any dissolved analyte. The intensity of the transient signal from a single particle, after subtraction of the dissolved signal intensity, is proportional to the number of atoms in the particle which can be converted to element mass and thus diameter to the third power, assuming a spherical particle shape. The number of pulses counted is proportional to the nanoparticle number concentration. The intensity of the continuum signal provides a measure of the dissolved metal content. The full width (at $10 \%$ peak height) of the transient signal from a single particle produced in an ICP-MS has been measured to be on the order of $0.34 \mathrm{~ms}$ [3]. When using dwell times that are significantly longer than the transient signal from a single particle, it is important that only one particle is detected per measurement period; coincident particles would result in a bias. Particle coincidence can be minimized by proper selection of the dwell time and by dilution of the sample. If a particle event is split between adjacent measurement periods, the intensities in each measurement period must be summed.

Samples are introduced into the ICP-MS as an aqueous suspension. Only a small percentage of the sample solution is transported into the plasma. The ratio of the amount of sample entering the plasma to the amount of sample introduced into the instrument is called the transport efficiency. Three calibration strategies are currently employed to quantify particle size and number concentration by spICP-MS: 1) Calibration of the instrument response using reference nanoparticle standards spanning the linear mass/size range of interest [4]. 2) Calibration of the instrument response using micro droplet generation [5], and 3) Measurement of the transport efficiency followed by calibration of the instrument response with ionic standard solution calibrants [6]. The last calibration strategy in this list is employed in this protocol. Calibration of the ionic mass fraction concentration is accomplished by measuring ionic standard solution calibrants.

Detection limits will depend on the elemental composition, the dissolved analyte content, and the sensitivity of the particular commercial instrument being used. For chemically homogenous NPs composed of a monoisotopic element (i.e., Au) and containing low mass 
concentration of ions, diameters in the size range of $10 \mathrm{~nm}$ to $200 \mathrm{~nm}$ can be measured and counted, but operating conditions may need to be adjusted to achieve a dynamic size range that is linear [7].

\section{Terminology}

Analysis time (t): Total measurement time per sample, e.g. $60 \mathrm{~s}$ to $6 \mathrm{~min}$

Dwell time $\left(t_{\text {dwell }}\right):$ The period during which the detector collects and integrates the analytical signal (measurement window), e.g. $0.05 \mathrm{~ms}$ to $10 \mathrm{~ms}$

Inductively Coupled Plasma Mass Spectrometry (ICP-MS): analytical technique used to measure the elemental and/or isotopic composition of a sample, based on an instrument comprising a sample introduction system, an inductively coupled plasma source for generation of ions of the material(s) under investigation, a plasma/vacuum interface, and a mass spectrometer comprising an ion focusing, separation and detection system [8].

Matrix: The majority component of a solution.

Nanoparticle: nano-object with all three external dimensions in the nanoscale [9].

Nanoparticle flux $\left(f_{\mathrm{NP}}\right)$ : The number of nanoparticles entering the plasma per unit of time, e.g., $\mathrm{s}^{-1}$

Nanoparticle number concentration $\left(N_{\mathrm{P}}\right)$ : The number of nanoparticles per volume or mass of solution, e.g., $\mathrm{mL}^{-1}, \mathrm{~L}^{-1}, \mathrm{~g}^{-1}, \mathrm{~kg}^{-1}$

Transport Efficiency $\left(\eta_{n}\right)$ : ratio of the number of particles $o r$ the mass of sample solution entering the plasma to the number of particles or mass of sample solution introduced into the instrument

Sample solution flow rate $\left(q_{\text {liq }}\right)$ : The mass or volume of sample solution introduced into the instrument per unit of time, e.g., $\mathrm{g} \cdot \mathrm{min}^{-1}, \mathrm{~mL} \cdot \mathrm{min}^{-1}$

4. Reagents, Materials, and Equipment

\subsection{Reagents}

4.1.1 De-ionized (DI) water ( $\geq 18 \mathrm{M} \Omega \cdot \mathrm{cm}$ resistivity)

Note: To achieve ultra-high purity (UHP) water, the DI feedstock water is subjected to subboiling distillation in-house.

4.1.2 UHP Nitric Acid, $\mathrm{HNO}_{3}$ (Optima Grade, Fisher Scientific, Pittsburg, PA)

4.1.3 UHP Hydrochloric Acid, $\mathrm{HCl}$ (Optima Grade, Fisher Scientific, Pittsburg, PA)

4.1.4 Thiourea (crystalline, ACS grade, $99 \%$, Alfa Aesar, Ward Hill, MA)

\subsection{Materials}


4.2.1 Reference Material (RM) 8011 Gold Nanoparticles, Nominal 10 nm Diameter (NIST, Gaithersburg, MD)

4.2.2 RM 8012 Gold Nanoparticles, Nominal $30 \mathrm{~nm}$ Diameter (NIST, Gaithersburg, MD)

4.2.3 RM 8013 Gold Nanoparticles, Nominal 60 nm Diameter (NIST, Gaithersburg, MD)

4.2.4 Standard Reference Material (SRM) 3121 Gold (Au) Standard Solution (NIST, Gaithersburg, MD)

4.2.5 SRM 3151 Silver (Ag) Standard Solution (NIST, Gaithersburg, MD)

4.2.6 RM 8017 PVP-Coated Silver Nanoparticles - Nominal Diameter $75 \mathrm{~nm}$ (NIST, Gaithersburg, MD)

\subsection{Labware}

4.3.1 Fluorinated Ethylene-Propylene (FEP) or perfluoroalkoxy (PFA) Teflon 1-L bottles

4.3.2 Nalgene low density polyethylene (LDPE) (30, 60, and 125) mL bottles

4.3.3 Falcon polypropylene $15 \mathrm{~mL}$ and $50 \mathrm{~mL}$ centrifuge tubes

4.3.4 High density polyethylene (HDPE) $4 \mathrm{~mL}$ scintillation vials

\subsection{Equipment}

4.4.1 ICP-MS capable of $t_{\text {dwell }} \leq 10 \mathrm{~ms}$

4.4.2 Analytical balance with readability of $0.1 \mathrm{mg}$

4.4.3 Ultrasonic bath

5. Reagent Preparation

5.1 Thiourea Diluent for Au standard solution

To improve the chemical stability, stability of the ICP-MS signal profile, and wash out characteristics of dilute solutions of ionic $\mathrm{Au}$, a diluent composed of 0.5 $\%$ mass fraction thiourea, $2.4 \%$ volume fraction $\mathrm{HCl}$, and $0.04 \%$ volume fraction $\mathrm{HNO}_{3}$ is used. Volume fraction is defined here as the volume of the constituent divided by the total volume of the solution. The response factor for ionic Au was tested in the thiourea diluent vs. water and found to be less than $5 \%$ different.

5.1.1 Add 5.00 g of crystalline thiourea to a 1-L, tared, clean PFA or FEP Teflon bottle followed by $500 \mathrm{~g}$ of UHP water. Cap and mix to dissolve the thiourea.

5.1.2 Add 24.00 mL (28.32 g) UHP HCl. Cap and mix.

5.1.3 Add $0.40 \mathrm{~mL}(0.56 \mathrm{~g})$ UHP $\mathrm{HNO}_{3}$.

5.1.4 Dilute to a final volume of $1.00 \mathrm{~L}(998.20 \mathrm{~g})$ with UHP water. Cap and mix.

5.2 Dilute $\mathrm{HNO}_{3}$ solution (2\% volume fraction)

Dilute $\mathrm{HNO}_{3}$ solution is used as a rinse between samples during ICP-MS analysis and in the preparation of dilute ionic silver standard solutions. Improvement in the chemical stability, stability of the ICP-MS signal profile, and wash out characteristics for $\mathrm{Ag}$ were obtained in dilute acid relative to water alone. On average, the response factor for ionic $\mathrm{Ag}$ in $2 \%$ volume fraction $\mathrm{HNO}_{3}$ solution was $10 \%$ higher than the response factor for ionic $\mathrm{Ag}$ in water though it is difficult to determine if the observed difference is due to a matrix effect in the ICP-MS or loss of Ag. 


\subsubsection{Add $40 \mathrm{~mL}$ (56.00 g) $\mathrm{UHP}_{\mathrm{HNO}}$ to $1 \mathrm{~L}$ UHP water and dilute to $2 \mathrm{~L}$ $(1996.40 \mathrm{~g})$ with UHP water.}

6. Preparation of Ionic Standard Solution Calibrants

Ionic standard solution calibrants are needed to calibrate the response of the instrument. The mass fractions given in Tables 1 and 2 serve as a guide and should be adjusted depending on the sensitivity of the instrument. Mass fractions with resulting count rates that span the range from 1.0E05 counts per second (cps) to $9.5 \mathrm{E} 05 \mathrm{cps}$ are recommended. A balance with a readability of $0.00001 \mathrm{~g}$ is used to record the masses for each of the dilution steps.

\subsection{Ionic Au Standard Solution Calibrants}

Au standard solution calibrants prepared in thiourea diluent are stable for 1 month.

6.1.1 Au Stock Solution, Dilution $1\left(A u D i l_{1}\right)$, nominal 1.00E $+05 \mathrm{ng} / \mathrm{g} \mathrm{Au}$

6.1.1.1 Record the mass of a clean, dry $60 \mathrm{~mL}$ LDPE bottle, $A u B_{1}$

6.1.1.2 Add 0.5 g of SRM 3121 Gold (Au) Standard Solution to the bottle and record the mass of the bottle + SRM 3121, $A u S_{1}$.

6.1.1.3 Dilute to $50 \mathrm{~g}$ with thiourea diluent and record the mass of the bottle + SRM 3121 + thiourea diluent, $A u D_{1}$

6.1.1.4 Calculate the exact mass fraction of ionic Au in Dilution 1:

$$
\mu \mathrm{g} / \mathrm{g} A u \mathrm{Dil}_{1}=\frac{\frac{m g}{g} S R M 3121 \times\left(A u S_{1}-A u B_{1}\right) \times 1,000}{A u D_{1}-A u B_{1}}
$$

6.1.2 Au Stock Solution, Dilution $2\left(A u D_{i l}\right)$, nominal 1,000 ng/g Au

6.1.2.1 Record the mass of a clean, dry $60 \mathrm{~mL}$ LDPE bottle, $A u B_{2}$

6.1.2.2 Add 0.5 $\mathrm{g}$ of Dilution 1 to the bottle and record the mass of the bottle + Dilution $1, \mathrm{AuS}_{2}$

6.1.2.3 Dilute to $50 \mathrm{~g}$ with thiourea diluent and record the mass of the bottle + Dilution $1+$ thiourea diluent, $A u D_{2}$

6.1.2.4 Calculate the exact mass fraction of ionic Au in Dilution 2:

$$
\mathrm{ng} / \mathrm{g} A u \mathrm{Dil}_{2}=\frac{\mu \mathrm{g} / \mathrm{g} A u D i l_{1} \times\left(A u S_{2}-A u B_{2}\right) \times 1,000}{A u D_{2}-A u B_{2}}
$$

6.1.3 Working Au Standards, nominal $5 \mathrm{ng} / \mathrm{g} \mathrm{Au}, 15 \mathrm{ng} / \mathrm{g} \mathrm{Au}, 30 \mathrm{ng} / \mathrm{g} \mathrm{Au}$ 6.1.3.1 In the same manner as described above, prepare working Au standards from $\mathrm{Au} \mathrm{Dil}_{2}$ according to the Table 1 . Three working standards are minimum; additional standards can be prepared. 
Table 1. Preparation of Working Au Standards

\begin{tabular}{|c|c|c|}
\hline $\begin{array}{c}\text { Nominal mass (g) of } A u \\
D i l_{2}\end{array}$ & $\begin{array}{c}\text { Nominal mass }(\mathrm{g}) \text { of } A u \\
D i l_{2}+\text { thiourea diluent }\end{array}$ & $\begin{array}{c}\text { Nominal mass fraction } \\
(\mathrm{ng} / \mathrm{g}) \mathrm{Au} \mathrm{Di}_{3}\end{array}$ \\
\hline 0.25 & 50 & 5 \\
\hline 0.75 & 50 & 15 \\
\hline 1.50 & 50 & 30 \\
\hline
\end{tabular}

6.2 Ionic Silver (Ag) Standard Solution Calibrants

To improve the stability of dilute solutions of ionic Ag and improve signal stability in the ICP-MS, Ag standards solutions are prepared in $2 \%$ volume fraction $\mathrm{HNO}_{3}$.

6.2.1 Ag Stock Solution, Dilution 1, nominal 1.00E+05 ng/g Ag

6.2.1.1 Using SRM 3151 Silver (Ag) Standard Solution, prepare in same manner as described in 6.1.1, except use $2 \%$ volume fraction $\mathrm{HNO}_{3}$ in place of the thiourea diluent.

6.2.2 Ag Stock Solution, Dilution 2, nominal 1,000 ng/g Ag

6.2.2.1 Using Ag stock solution, Dilution 1, prepare in same manner as described in 6.1.2, except use $2 \%$ volume fraction $\mathrm{HNO}_{3}$ in place of the thiourea diluent.

6.2.3 Working Ag Standards, nominal $2 \mathrm{ng} / \mathrm{g} \mathrm{Ag}, 5 \mathrm{ng} / \mathrm{g} \mathrm{Ag}, 8 \mathrm{ng} / \mathrm{g} \mathrm{Au}$

6.2.3.1 In the same manner as described above, prepare working Ag standards from $\mathrm{Ag} \mathrm{Dil} l_{2}$ according to the Table 2. Three working standards are minimum; additional standards can be prepared.

Table 2. Preparation of Working Ag Standards

\begin{tabular}{|c|c|c|}
\hline $\begin{array}{c}\text { Nominal mass }(\mathbf{g}) \text { of } \\
\mathbf{A g ~} \mathrm{Dil}_{\mathbf{2}}\end{array}$ & $\begin{array}{c}\text { Nominal mass (g) of } \boldsymbol{A u} \\
\mathbf{D i l}_{\mathbf{2}}+\mathbf{2} \% \text { volume } \\
\text { fraction } \mathbf{H N O}_{\mathbf{3}} \text { diluent }\end{array}$ & $\begin{array}{c}\text { Nominal mass fraction } \\
(\mathbf{n g} / \mathbf{g}) \mathbf{A g ~ D i l}_{\mathbf{3}}\end{array}$ \\
\hline 0.12 & 60 & 2 \\
\hline 0.30 & 60 & 5 \\
\hline 0.48 & 60 & 8 \\
\hline
\end{tabular}

7. Sample Preparation

Proper dilution of the NP suspension prior to spICP-MS analysis is important. The intent is to avoid, on one hand, the bias associated with more than one particle event occurring per $t_{\text {dwell }}$ while at the same time maximizing the number of measured events occurring during the total acquisition time, $t$. The first step is to calculate the target particle flux.

7.1 Determination of Target Particle Flux for spICP-MS Analysis

Laborda et al. suggest that the number of nanoparticles entering the ICP per unit of time, i.e., the particle flux, $f_{\mathrm{NP}}$ be calculated at the point where the systematic error in the number of counted events arising from counting a 2-nanoparticle event as a single nanoparticle event is approximately equal to the random error of the measurement (derived by Poisson statistics from the total number of counted nanoparticle events) [10]. From [10], the systematic error, bias $_{\mathrm{NP}}$ due to 2-nanoparticle events which are counted as one nanoparticle can be expressed as: 


$$
\text { bias }_{N P} \approx \frac{t_{d w e l l}}{2} \times f_{N P}
$$

The relative standard deviation (RSD) of the measurement, $\mathrm{RSD}_{\mathrm{NP}}$ is governed by Poisson statistics and is derived from the analysis time, $t$ and particle flux, $f_{\mathrm{NP}}$ as follows:

$$
\mathrm{RSD}_{\mathrm{NP}}=\frac{1}{\sqrt{t \times f_{N P}}}
$$

The particle flux at which both error sources are equal can be derived by setting equation 1 equal to equation 2 , and solving for $f_{\mathrm{NP}}$ as follows:

$$
f_{\mathrm{NP}}=\sqrt[3]{\frac{4}{t \times t_{d w e l l^{2}}}}
$$

Thus, the target particle flux will depend on the chosen dwell and analysis times. Equation 3 implies that for a given analysis time, $t$, smaller $t_{\text {dwell }}$ result in a higher

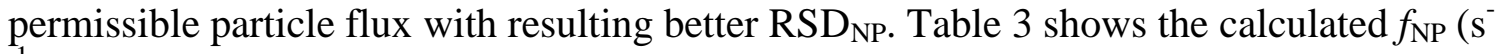
${ }^{1}$ ) and $\mathrm{RSD}_{\mathrm{NP}}$ for an analysis time of $60 \mathrm{~s}$ and dwell times ranging from $1 \mathrm{~ms}$ to $10 \mathrm{~ms}$.

Table 3. Calculated particle flux, $f_{\mathrm{NP}}\left(\mathrm{s}^{-1}\right)$ and theoretical measurement precision, $\mathrm{RSD}_{\mathrm{NP}}$ for analysis time, $t$ of $60 \mathrm{~s}$ and dwell times, $t_{\mathrm{dwell}}$ ranging from 0.01 ms to $10 \mathrm{~ms}$

\begin{tabular}{|c|c|c|}
\hline $\boldsymbol{t}_{\text {dwell }}(\mathbf{m s})$ & $\boldsymbol{f}_{\mathbf{N P}}\left(\mathbf{s}^{-\mathbf{1}}\right)$ & $\mathbf{R S D}_{\mathbf{N P}}$ \\
\hline 10 & 9 & 0.044 \\
\hline 5 & 14 & 0.035 \\
\hline 3 & 20 & 0.029 \\
\hline 1 & 41 & 0.020 \\
\hline 0.1 & 188 & 0.0094 \\
\hline 0.05 & 299 & 0.0075 \\
\hline 0.01 & 874 & 0.0041 \\
\hline
\end{tabular}

However, there is a practical limit to be considered here. As the dwell time decreases the likelihood that the nanoparticle event is split over adjacent measurement windows increases. Liu et al. found that for data collected at a dwell time of $1 \mathrm{~ms}$, over $40 \%$ of the particle events were spilt between adjacent measurement windows, and accurate analysis required reconstruction of the NP event through additional data manipulation [7]. Currently, only a few commercially available ICP-MS systems provide software to count and size NP events that are split over adjacent measurement windows. In light of these competing considerations and when specialized software is not used, our protocol is to use a dwell time of $10 \mathrm{~ms}$, where the probability of a split event is reduced to $4 \%$, and to improve the precision of the measurement by using longer analysis times. Table 4 shows the calculated $f_{\mathrm{NP}}\left(\mathrm{s}^{-1}\right)$ and $\mathrm{RSD}_{\mathrm{NP}}$ for a dwell time of $10 \mathrm{~ms}$ and analysis times ranging from $60 \mathrm{~s}$ to $360 \mathrm{~s}$. 
Table 4. Calculated particle flux, $f_{\mathrm{NP}}\left(\mathrm{sec}^{-1}\right)$ and theoretical measurement precision, $\mathbf{R S D}_{\mathrm{NP}}$ for dwell time, $t_{\mathrm{dwell}}$ of $10 \mathrm{~ms}$ and analysis times, $t$ ranging from $60 \mathrm{~s}$ to $360 \mathrm{~s}$

\begin{tabular}{|c|c|c|}
\hline $\boldsymbol{t}(\mathbf{s})$ & $\boldsymbol{f}_{\mathbf{N P}}\left(\mathbf{s}^{-\mathbf{1}}\right)$ & $\mathbf{R S D}_{\mathbf{N P}}$ \\
\hline 60 & 9 & 0.044 \\
\hline 120 & 7 & 0.035 \\
\hline 240 & 6 & 0.028 \\
\hline 360 & 5 & 0.024 \\
\hline
\end{tabular}

The values listed in Table 4 are used as an upper bound on the target particle flux.

7.2 Calculation of the Particle Number Concentration, $N_{\mathrm{NP}}$ from the Target Particle Flux

Once the target particle flux is determined, the particle number concentration $\left(N_{\mathrm{NP}}\right)$ of the diluted sample that will yield the desired flux can be calculated from the transport efficiency $\left(\eta_{\mathrm{n}}\right)$ and the sample solution flow rate $\left(q_{\text {liq }}\right)$. Since calculation of the target particle number concentration requires prior knowledge of the transport efficiency, and determination of the transport efficiency requires measurement of a properly diluted nanoparticle suspension, this will be an iterative process.

The target particle number concentration can be calculated as follows:

$$
N_{N P}=\frac{f_{N P} \times 60}{q_{l i q} \times \eta_{n} / 100}
$$

where $N_{\mathrm{NP}}$ is the target particle number concentration $\left(\mathrm{g}^{-1}\right), f_{\mathrm{NP}}$ is the target particle flux $\left(\mathrm{s}^{-1}\right), q_{\text {liq }}$ is the sample solution flow rate $\left(\mathrm{g} \cdot \mathrm{min}^{-1}\right)$, and $\eta_{\mathrm{n}}$ is the transport efficiency expressed as a percentage. Table 5 shows the calculated particle number concentration for different particle flux, sample solution flow rates and transport efficiencies.

Table 5. Calculated particle number concentration, $\mathbf{N}_{\mathrm{NP}}$ for varying particle flux, $f_{\mathrm{NP}}$, sample solution flow rate, $q_{\text {liq }}$, and transport efficiency, $\eta_{\mathbf{n}}$

\begin{tabular}{|c|c|c|c|}
\hline $\boldsymbol{f}_{\mathbf{N P}}\left(\mathbf{s}^{-\mathbf{1}}\right)$ & $\boldsymbol{q}_{\text {liq }}\left(\mathrm{g} \cdot \mathrm{min}^{-1}\right)$ & $\boldsymbol{\%} \boldsymbol{\eta}_{\mathbf{n}}$ & $\boldsymbol{N}_{\mathbf{N p}}\left(\mathbf{g}^{-\mathbf{1}}\right)$ \\
\hline 5 & 1 & 4 & 7,500 \\
\hline 9 & 1 & 4 & 13,500 \\
\hline 5 & 1 & 2 & 15,000 \\
\hline 9 & 1 & 2 & 27,000 \\
\hline 5 & 0.2 & 4 & 37,500 \\
\hline 9 & 0.2 & 4 & 67,500 \\
\hline 5 & 0.2 & 2 & 75,000 \\
\hline 9 & 0.2 & 2 & 135,000 \\
\hline \hline
\end{tabular}

7.3 Calculation of the Particle Number Concentration From Known Mass Concentration and Particle Size 
Often, little is known about the particle number concentration of a sample suspension. However, with knowledge of the total mass concentration (assuming all analyte present is in nanoparticle form) and the expected particle size of the sample, the particle number concentration can be calculated as follows:

$$
N_{N P}=\frac{6 \times C_{S}}{1 E^{-15} \times d_{p}{ }^{3} \times \pi \times \rho}
$$

where $N_{\mathrm{NP}}$ is the target particle number concentration $\left(\mathrm{g}^{-1}\right), C_{\mathrm{S}}$ is the mass fraction of the analyte in the sample $\left(\mu \mathrm{g} \cdot \mathrm{g}^{-1}\right), d_{\mathrm{p}}$ is the diameter of the particle $(\mathrm{nm}), \pi$ is pi and $\rho$ is the density of the particle $\left(\mathrm{g} \cdot \mathrm{cm}^{-3}\right)$. Table 6 lists the calculated particle number concentration for NIST nanoparticle RMs value assigned for mass fraction and nanoparticle size. For consistency, the particle size measured by transmission electron microscopy (TEM) is used.

Table 6. Calculated particle number concentration, $N_{\mathrm{NP}}$ for NIST nanoparticle RMs value assigned for mass fraction and nanoparticle size

\begin{tabular}{|c|c|c|c|c|}
\hline RM & $\mathbf{8 0 1 1}$ & $\mathbf{8 0 1 2}$ & $\mathbf{8 0 1 3}$ & $\mathbf{8 0 1 7}$ \\
\hline Composition & $\mathrm{Au}$ & $\mathrm{Au}$ & $\mathrm{Au}$ & $\mathrm{Ag}$ \\
\hline density $^{\mathrm{a}}$ & 19.3 & 19.3 & 19.3 & 10.49 \\
\hline$C_{\mathrm{S}}\left(\mu \mathrm{g}^{-\mathrm{g}^{-1}}\right)$ & 51.56 & 48.17 & 51.86 & $1,081^{\mathrm{b}}$ \\
\hline TEM diameter $(\mathrm{nm})$ & 8.9 & 27.6 & 56 & 74.6 \\
\hline Calculated $\boldsymbol{N}_{\mathbf{N P}}\left(\mathbf{g}^{-1}\right)^{\mathbf{c}}$ & $\mathbf{7 . 2 3 7 E}+\mathbf{1 2}$ & $\mathbf{2 . 2 6 7 E + 1 1}$ & $\mathbf{2 . 9 2 2 E}+\mathbf{1 0}$ & $\mathbf{4 . 7 4 1 E}+\mathbf{1 1}$ \\
\hline
\end{tabular}

a of bulk metal; ${ }^{b}$ assumes reconstitution with $2.000 \mathrm{~g}$ water, ${ }^{\mathrm{c}}$ based on assumption that all analyte is present as spherical nanoparticles of the listed TEM - measured diameter.

\subsection{Preparation of Working Nanoparticle Suspensions of NIST RMs}

NIST RMs must be serially diluted to obtain working suspensions with particle number concentrations suitable for spICP-MS analysis. Table 7 shows a possible dilution scheme for RMs 8011, 8012, 8013, and 8017 to achieve a particle number concentration of $3.0 \mathrm{E}+04 \mathrm{~g} \mathrm{~g}^{-1}$. In general, high purity water is used as the diluent in the preparation of these suspensions, but for reactive elements such as Ag, care must be exercised and alternative diluents may be useful for stabilizing the suspension. Clean, dry, $60 \mathrm{~mL}$ and $125 \mathrm{~mL}$ LDPE bottles or $50 \mathrm{~mL}$ polypropylene centrifuge tubes are used as preparation vessels. A balance with a readability of $0.00001 \mathrm{~g}$ is used to record the masses for each of the dilutions steps. When preparing NP suspensions, it is advisable to add the diluent to the bottle first followed by the aliquot of the suspension being diluted. Pipet tips should be conditioned with the suspension prior to aliquoting. Each diluted suspension is sonicated for one minute in an ultrasonic bath. Nominal mass fractions $\left(C_{\mathrm{S}}\right)$ of each dilution are listed in Table 7.

Table 7. Preparation of Working Nanoparticle Suspensions of NIST RMs

\begin{tabular}{|c|c|c|c|c|}
\hline RM & $\mathbf{8 0 1 1}$ & $\mathbf{8 0 1 2}$ & $\mathbf{8 0 1 3}$ & $\mathbf{8 0 1 7}$ \\
\hline \multicolumn{5}{|c|}{ Dilution 1 $\mathbf{1}^{\mathbf{a}, \mathbf{b}}$} \\
\hline mass (g) of RM & 0.200 & 0.250 & 0.500 & 0.200 \\
\hline mass (g) RM + water & 125 & 50.0 & 50.0 & 50.0 \\
\hline
\end{tabular}




\begin{tabular}{|c|c|c|c|c|}
\hline$C_{\mathrm{S}} \operatorname{Dil}_{1}(\mathrm{ng} / \mathrm{g})$ & 82.5 & 241 & 519 & 4324 \\
\hline$N_{\mathrm{NP}} \operatorname{Dil}_{1}\left(\mathrm{~g}^{-1}\right)$ & $1.16 \mathrm{E}+10$ & $1.13 E+09$ & $2.92 \mathrm{E}+08$ & $1.90 \mathrm{E}+09$ \\
\hline \multicolumn{5}{|c|}{ Dilution 2} \\
\hline mass $(\mathrm{g})$ of $\mathrm{Dil}_{1}$ & 0.200 & 0.250 & 0.500 & 0.200 \\
\hline mass $(\mathrm{g}) D i l_{1}+$ water & 125 & 50.0 & 50.0 & 50.0 \\
\hline$C_{\mathrm{S}} \mathrm{Dil}_{2}(\mathrm{ng} / \mathrm{g})$ & 0.132 & 1.20 & 5.19 & 17.30 \\
\hline$N_{\mathrm{NP}} \mathrm{Dil}_{2}\left(\mathrm{~g}^{-1}\right)$ & $1.85 \mathrm{E}+07$ & $5.67 \mathrm{E}+06$ & $2.92 \mathrm{E}+06$ & $7.58 \mathrm{E}+06$ \\
\hline \multicolumn{5}{|c|}{ Dilution 3} \\
\hline mass $(\mathrm{g})$ of $\mathrm{Dil}_{2}$ & 0.203 & 0.265 & 0.514 & 0.198 \\
\hline mass $(\mathrm{g}) \mathrm{Dil}_{2}+$ water & 125 & 50.0 & 50.0 & 50.0 \\
\hline$C_{\mathrm{S}} \mathrm{Dil}_{3}(\mathrm{ng} / \mathrm{g})$ & 0.000214 & 0.00638 & 0.0533 & 0.0685 \\
\hline$N_{\mathrm{NP}} \mathrm{Dil}_{3}\left(\mathrm{~g}^{-1}\right)$ & $3.01 \mathrm{E}+04$ & $3.00 \mathrm{E}+04$ & $3.00 \mathrm{E}+04$ & $3.00 \mathrm{E}+04$ \\
\hline
\end{tabular}

${ }^{a}$ Note 1: For RMs 8011 - 8012: Thoroughly mix the vial by inverting several times, do not sonicate the starting material. Inspect for proper appearance. Dilute suspensions of AuNPs should be prepared daily.

bote 2: For RM 8017: Reconstitute RM 8017 per the instructions on the Report of Investigation (ROI) using $2.000 \mathrm{~g}$ water. Do not sonicate the reconstituted suspension. Data on the stability of reconstituted RM 8017 stored at $4{ }^{\circ} \mathrm{C}$ shows minimal degradation of the Ag nanoparticles over a period of $90 \mathrm{~d}$, but dilute suspensions of AgNPs prepared in water are unstable. Dilutions should be performed the day of analysis, and the working suspension should be analyzed within one half hour of preparation if water is used as the diluent.

8. ICP-MS Analysis

Any ICP-MS system that can be operated in time-resolved analysis mode (TRA) and is capable of $t_{\text {dwell }} \leq 10 \mathrm{~ms}$ can be used for spICP-MS analysis. A ThermoFisher X series 2 quadrupole ICP-MS system was used to collect the data reported in this protocol. The plasma is operated at a forward power of $1400 \mathrm{~W}$, and the plasma and auxiliary flows are set to $14 \mathrm{~L} \cdot \mathrm{min}^{-1}$ and $0.9 \mathrm{~L} \cdot \mathrm{min}^{-1}$, respectively. The nebulizer flow is adjusted daily to achieve optimum conditions, but is typically between $0.8 \mathrm{~L} \cdot \mathrm{min}^{-1}$ and $0.9 \mathrm{~L} \cdot \mathrm{min}^{-1}$. Sample solutions are introduced into the ICP via a peristaltic pump and a micro-flow perfluoroalkoxy (PFA) concentric nebulizer (PFA-ST, Elemental Scientific, Omaha, NE, USA) attached to an impact bead spray chamber cooled to $2{ }^{\circ} \mathrm{C}$. The sample solution flow rate $\left(q_{\text {liq }}\right)$, nominally $0.2 \mathrm{~g} \cdot \mathrm{min}^{-1}$, is measured daily in triplicate. The transport efficiency, which will vary with the sample introduction system (i.e., type of nebulizer and spray chamber), operating conditions, (i.e., gas flows and cone type), and sample matrix, is measured daily using both the particle frequency method and the particle size method reported in Pace et al. [6]

8.1 Optimization of operating conditions

Operating conditions are optimized per manufacturer recommendations. A tune solution containing lithium ( $\mathrm{Li})$, indium (In), cerium (Ce) and uranium (U) is used; nebulizer gas flow and lens settings are adjusted to obtain maximum sensitivity for ${ }^{115} \mathrm{In}$ and then further adjusted until an acceptable ${ }^{156} \mathrm{CeO}^{+} /{ }^{140} \mathrm{Ce}^{+}$ ratio is obtained. Gold and $\mathrm{Ag}$ are intentionally absent from the tune solution in order to keep the instrument background low for those elements. ThermoFisher X 
series ICP-MS instruments are supplied with two skimmer cone types, the matrix tolerant Xt skimmer cone, and the higher sensitivity, Xs cone. The Xs cone provides the best detection limits; $\geq 700$ cps can be obtained for $10 \mathrm{~nm}$ AuNPs, but at the expense of the ${ }^{156} \mathrm{CeO}^{+} /{ }^{140} \mathrm{Ce}^{+}$ratio, which is typically $6 \%$ to $15 \%$ for the chosen tune conditions. Due to the greater sensitivity for the Xs cones, the upper size range is limited to about $60 \mathrm{~nm}$ AuNPs. Particles larger than this may exceed the linear dynamic range of the pulse counting detector since the signal intensity for a spherical particle scales by a power of three relative to the particle diameter. Though a linear relationship between the pulse stage and analog stage of the detector can be achieved for ionic standards, our experience with particulate suspensions is that non- linearity is observed for suspensions containing large particles whose signal intensity exceeds the range of the pulse detector. Reducing sensitivity will extend the measureable size range. The Xt cones are used for routine analysis; conditions are chosen so the ${ }^{156} \mathrm{CeO}^{+} /{ }^{140} \mathrm{Ce}^{+}$ ratio is $<2 \%$, and AuNPs in the range of $20 \mathrm{~nm}$ to $80 \mathrm{~nm}$ can be measured. The upper measurable size range can be extended by lowering the signal intensity using collision cell/kinetic energy discrimination mode or by lowering the extraction voltage [7]. As the Ag isotopes are half as abundant as the Au isotope, the lower size limit is $20 \mathrm{~nm}$ for AgNPs using the Xs cones, and the upper limit is $100 \mathrm{~nm}$ AgNPs with Xt cones under standard conditions. Different ICP-MS systems will have different lower and upper size detection limits depending on the sensitivity of the system.

8.2 Analysis Parameters

8.2.1 Au: The ${ }^{197} \mathrm{Au}$ intensity is recorded in TRA mode using a dwell time of $10 \mathrm{~ms}$ for total analysis times $(t)$ ranging from $100 \mathrm{~s}$ to $360 \mathrm{~s}$.

8.2.2 Ag: The ${ }^{107} \mathrm{Ag}$ intensity is recorded in TRA mode using a dwell time of $10 \mathrm{~ms}$ for total analysis times $(t)$ ranging from $100 \mathrm{~s}$ to $360 \mathrm{~s}$.

8.3 Measurement of the Transport Efficiency

Measurement of the transport efficiency via the methods outlined in Pace et al., the particle frequency method and the particle size method, requires measurement of the sample flow, analysis of ionic standards (size method), and analysis of a standard nanoparticle suspension of known size and mass concentration or particle number concentration [6] . Calibration of the transport efficiency in this way means that the overall uncertainty of the spICP-MS measurement of size and number concentration is directly related to the measurement method and its associated uncertainty used to value assign the standard nanoparticle suspension. We use RM 8013 and the size value assigned by TEM, $56 \mathrm{~nm} \pm 0.5 \mathrm{~nm}$. The derived particle number concentration for RM 8013 is shown in Table 6, and preparation of the working suspension is described in section 7.4.

Pace et al. reports similar values for the transport efficiency measured using either the frequency or size method, but we have at times observed that the transport efficiency measured via the particle frequency method is lower than that measured by the particle size method. Loss of NPs to the container walls and pump tubing from the very dilute suspensions required for spICP-MS would result in a low bias in the transport efficiency measured via the particle frequency method and could possibly explain at least some of the differences we observe. 
Tuoriniemi et al. have also identified analyte partitioning effects during nebulization and off axis trajectories of particles in the plasma as other considerations that may affect the accurate measurement of the transport efficiency [11]. Our protocol is to measure the transport efficiency using both methods (described below), however, our experience has been that when a difference is observed, the particle size method yields more accurate results.

\subsubsection{Measurement and Calculation of sample flow rate, $q_{\text {liq }}$}

The sample flow is the amount of sample solution entering the instrument per unit of time.

8.3.1.1 Measure the total starting mass of the sample solution and its container using a balance with a readability of $0.0001 \mathrm{~g}$. Record as $m_{\text {start }}(\mathrm{g})$

8.3.1.2 Using a stop watch, measure the time from when the sample solution uptake starts to the point where the probe leaves the solution. Record as $t_{\text {uptake }}(\mathrm{min})$

8.3.1.3 Measure and record the mass of the remaining sample solution and its container $m_{\mathrm{end}}(\mathrm{g})$

8.3.1.4 Calculate the sample solution flow rate $\left(q_{\text {liq }}\right)$ as:

sample solution flow rate $\left(g \cdot \min ^{-1}\right)=\frac{m_{\text {end }}-m_{\text {start }}}{t_{\text {uptake }}}$

\subsubsection{Analysis sequence for the measurement of the transport efficiency}

8.3.2.1 Aspirate thiourea solution until instrument background at $\mathrm{m} / \mathrm{z} 197$ is at lowest achievable level.

8.3.2.2 Run samples in the following run sequence: thiourea diluent, water, NP reference material (i.e., RM 8013 - D-3, see Table 7), working Au standards from low to high concentration. If desired a second NP reference material (i.e., RM 8012 - Dil3) can be run and used as an accuracy check. At least three replicates of the NP reference material are measured and the results averaged.

\subsubsection{Data Analysis}

8.3.3.1 Using a spread sheet program such as Microsoft Excel, multiply the data recorded in counts per second by the dwell time, $t_{\mathrm{dwell}}$, in order to convert to counts per measurement window.

8.3.3.2 Distinguishing particle events from background

Particle events are distinguished from the background using a $n$ times standard deviation $(n \times \sigma)$ criterion as described below. Tuoriniemi et al. suggest that in order to reduce the number of false positives (signals counted as a particle, but which are not particles) to less than $0.1 \%$ of the total count, $n$ values larger than 3 must be used. They recommend a value of $n=5$ as a compromise between minimization of the number of false positives, while not omitting too many particles from being counted that are in fact particles.

8.3.3.2.1 Order the count data for each sample from largest to smallest 8.3.3.2.2 Compute the mean $(\mu)$, standard deviation $(\sigma)$, and $\mu+5 \times \sigma$ 
8.3.3.2.3 Using the value for $\mu+5 \times \sigma$ as the cutoff between particle signal and background, remove the particle signals from the population and move these data points to a different 'particle events' column.

8.3.3.2.4 Compute the mean $(\mu)$, standard deviation $(\sigma)$, and $\mu+5 \times \sigma$ of the remaining population of data points. If additional particle signals exceed the new value for $\mu+5 \times \sigma$, again remove the particle signals from the population and move these data points to the 'particle events' column. Continue this iterative process until none of the remaining data points exceeds $\mu+5 \times \sigma$.

8.3.3.2.5 Compute the mean of the data points not considered particle events, this is the intensity of the dissolved or ionic fraction, $I_{\text {diss }}$. Note that this population may also include particles that are too small to be detected as particles.

8.3.3.2.5.1 Correct $I_{\text {diss }}$ for instrument background by subtracting the mean of the population of data points measured for the water sample.

\subsubsection{Split Particle Correction}

The transport of particles into the plasma is a random process. It is possible that a particle event may occur near the end of one measurement period (dwell time) and spill over into the start of the adjacent measurement period. This is considered a split particle event. As discussed in section 7.1, when the chosen dwell time approaches the width of a single particle event, the likelihood that a particle event is spilt between adjacent measurement windows increases. For samples of known monodispersity, as should be the case for the NP standard being used to measure the transport efficiency, it is possible to correct for split particle events. Examples of split particle correction for various $t_{\text {dwell }}$ are given in a spreadsheet in the Supporting Information of [7] and will not be described in detail here. Briefly, the temporal data are examined to locate occurrences where signal was observed in adjacent measurement windows. The intensity of the signal in each adjacent measurement window is compared to the intensity that would be expected for a single particle event contained completely within one dwell time. If either or both of the observed intensities in the adjacent measurement windows are more than $25 \%$ lower than the expected intensity, then the signals are assumed to be from a single particle event and this split particle event is corrected by summing the two intensities.

\subsubsection{Visual inspection for false positives}

A false positive is a signal counted as a particle, but which is not a particle. Though the $n$ times standard deviation $(n \times \sigma)$ criterion described above is selected to reduce the number of false positives, some false positives remain in the data set. In situations where the measured suspension is believed to be monodisperse, contains particles well above the size detection limit, and contains little or no dissolved analyte, the data 
set can be visually examined for false positives. In this case, a large gap (greater than a factor of ten) will exist between the intensity of a true particle event and false positive events.

8.3.3.5 Compute the mean of the data points considered particle events corrected for split particle events and false positives. This is the intensity of the particle events, $I_{\mathrm{NP}}$.

8.3.3.5.1.1 Correct $I_{\mathrm{NP}}$ for the dissolved background by subtracting $I_{\text {diss }}$ (see 8.3.3.2.5).

8.3.3.6 Count the number of data points considered particle events corrected for split particle events and false positives $\left(\right.$ Count $\left._{\mathrm{NP}}\right)$

8.3.3.7 Compute the nanoparticle flux, $f_{\mathrm{NP}}\left(\mathrm{s}^{-1}\right)$

$$
f_{N P}\left(s^{-1}\right)=\frac{\text { Count }_{N P}}{t_{\text {analysis }}}
$$

where Count $_{\mathrm{NP}}$ is the number of particle events measured (see 8.3.3.6) during the analysis time ( $t$, sec, see 8.2.1).

8.3.4 Calculation of Transport Efficiency via the Particle Frequency Method, $\eta_{\mathrm{nPF}}$

$$
\eta_{n P F}=\frac{f_{N P}}{N_{N P R M D i l 3} \times q_{l i q} / 60}
$$

where $f_{\mathrm{NP}}\left(\mathrm{s}^{-1}\right)$ is the nanoparticle flux measured for the nanoparticle reference material, $N_{\mathrm{Np} \mathrm{RM} \mathrm{Dil3}}\left(\mathrm{g}^{-1}\right.$, Table 7$)$ is the calculated particle number concentration of the gravimetrically diluted nanoparticle reference material, and $q_{\text {liq }}$ is the sample flow $\left(\mathrm{g} \cdot \mathrm{min}^{-1}\right)$

8.3.5 Calculation of Transport Efficiency via the Particle Size Method, $\eta_{\mathrm{nPS}}$

8.3.5.1 Construct mass per measurement period $\left(t_{\mathrm{dwell}}\right)$ calibration curve for ionic standards to derive the ionic response factor, $R F_{\text {ionic }}$

8.3.5.1.1 Compute mean measured counts for working Au standards, $I_{\text {Au ionic }}$, and correct for instrument background by subtracting the mean of the population of data points measured for the thiourea diluent.

8.3.5.1.2 Compute the mass of dissolved Au standard (ng) introduced into the instrument per measurement period, $\operatorname{mass}_{\text {Au ionic }}$, from the mass concentrations of working standards (Table1):

$$
\operatorname{mass}_{\text {Au ionic }}(n g)=\frac{A u \mathrm{Dil}_{3} \times q_{\text {liq }} \times t_{d w e l l}}{6.0 \mathrm{E}^{+04}}
$$


where $\mathrm{Au} \mathrm{Dil} l_{3}$ is the mass fraction of ionic Au in the working standards (nominal $5 \mathrm{ng} / \mathrm{g}$ to $30 \mathrm{ng} / \mathrm{g} \mathrm{Au}$ ), $q_{\text {liq }}$ is the sample flow $\left(\mathrm{g} \cdot \mathrm{min}^{-1}\right), t_{\mathrm{dwell}}$ is the dwell time $(\mathrm{ms})$.

8.3.5.1.3 Plot $I_{\text {Au ionic }}$ Vs. mass $_{\text {Au ionic }}$ for each Au working standard and using the regression function of Microsoft Excel, compute the slope with units of counts $\mathrm{ng}^{-1}$, this is the ionic response factor, $R F_{\text {ionic. The }}$ regression function of Microsoft Excel also computes the standard error of the slope which can be used in the computation of the standard uncertainty of the transport efficiency measurement (see Uncertainty section).

8.3.5.2 Compute response factor $\left(R F_{\mathrm{NP}}\right)$ for NP standard

8.3.5.2.1 Compute the mass of the NP in the NP standard, mass $_{\mathrm{NP}}$ RM $(\mathrm{ng})$

$$
\operatorname{masS}_{N P R M}(n g)=\frac{d_{p R M}{ }^{3} \times \pi \times \rho \times 1 \mathrm{E}^{-12}}{6}
$$

where $d_{\mathrm{p} \mathrm{RM}}$ is the diameter of the particle $(\mathrm{nm})$ in the standard, $\pi$ is pi and $\rho$ is the density of the particle $\left(\mathrm{g} \cdot \mathrm{cm}^{-1}\right)$. For use of RM 8013 as the NP standard, the particle size measured by TEM, $56 \mathrm{~nm}$, is used (see section 8.3), and the calculated mass $_{\mathrm{NP} \text { RM }}$ is $1.775 \mathrm{E}$ $06 \mathrm{ng} \mathrm{Au}$.

8.3.5.2.2 Compute the response factor of the NP standard, $\mathrm{RF}_{\mathrm{NP} \mathrm{RM}}$

$$
R F_{N P R M}=\frac{I_{\mathrm{NP} \mathrm{RM}}-I_{\mathrm{diss} \mathrm{RM}}}{\operatorname{mass}_{\mathrm{NP} \mathrm{RM}}}
$$

where $I_{\mathrm{NP} \text { RM }}$ is the intensity of the particle events, $I_{\text {diss RM }}$ is the intensity of the dissolved background (counts, see 8.3.3.5) and mass $_{\mathrm{NP}} \mathrm{RM}$ is the mass Au in a single NP within the NP standard (ng, see 8.3.5.2.1).

8.3.5.3 Compute the Transport Efficiency (particle size method), $\eta_{\mathrm{nPS}}$

$$
\eta_{n P S}=\frac{R F_{\text {Au ionic }}}{R F_{N P R M}}
$$

where $R F_{\text {Au ionic }}$ is the slope of the calibration curve formed by a plot of $\mathrm{I}_{A u}$ ionic Vs. mass ${ }_{A u}$ ionic for each Au working standard (counts·ng ${ }^{-1}$, see 8.3.5.1.3) and $R F_{\mathrm{NP}}$ is the response factor of the NP standard (counts.ng ${ }^{-1}$, see 8.3.5.2).

\subsection{Measurement of 'Unknown' Ag Nanoparticle Suspension}

8.4.1 Dilute samples to target particle number concentration (See section 7) Note: Dilution of unknowns to the target particle number concentration requires a priori knowledge of the particle size and mass concentration of the NPs in the suspension. If this 
information is not available, a series of dilutions must be analyzed to demonstrate the absence of particle coincidence.

8.4.2 Aspirate $2 \%$ volume fraction nitric acid solution until instrument background at $\mathrm{m} / \mathrm{z} 107$ is at lowest achievable level.

8.4.3 Run samples in the following run sequence: $2 \%$ volume fraction nitric acid solution, water, unknowns from low $N_{\mathrm{NP}}$ to high $N_{\mathrm{NP}}$, working Ag standards from low to high concentration.

Note: Dilute suspensions of AgNPs are reactive and should be prepared within $30 \mathrm{~min}$ of analysis or prepared in a diluent that will stabilize the AgNPs.

8.4.4 Perform data analysis as described in section 8.3.3.

Note: Split particle correction and visual inspection for false positives may not be possible for the analysis of unknown suspensions.

9. Calculation of Particle Number Concentration of 'Unknown 'Suspension of AgNPs

$$
N_{N P \text { unknown }}=\frac{\text { Count }_{N P \text { unknown }}}{t_{\text {analysis }} \times q_{\text {liq }} / 60 \times \eta_{\mathrm{n}}} \times \text { Dilution Factor }
$$

where Count $_{\mathrm{NPunknown}}$ is the number of particle events measured during the analysis time, $t_{\text {analysis }}(\mathrm{s}), q_{\text {liq }}$ is the sample flow $\left(\mathrm{g} \cdot \mathrm{min}^{-1}\right)$, and $\eta_{\mathrm{n}}$ is the transport efficiency (section 8.3). Logically the transport efficiency measured via the particle frequency method, $\eta_{\mathrm{nPF}}$, can be used, but only if there is no significant difference between $\eta_{\mathrm{nPF}}$ and $\eta_{\mathrm{nPS}}$.

10. Calculation of Particle Mass, Particle Diameter, and Particle Size Distribution of 'Unknown' Suspension of AgNPs

10.1 Construct mass per measurement period calibration curve for ionic standards

10.1.1 Compute mean measured counts for working Ag standards, $I_{\mathrm{Ag} \text { ionic }}$, and correct for instrument background by subtracting the mean of the population of data points measured for the $2 \%$ volume fraction nitric acid diluent.

10.1.2 Compute the mass of dissolved Ag standard (ng) introduced into the

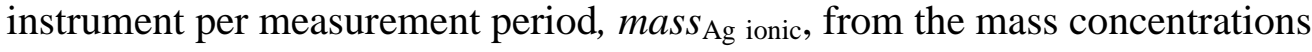
of working standards (Table 2):

$$
\operatorname{mass}_{\text {Ag ionic }}(n g)=\frac{A g \mathrm{Dil}_{3} \times q_{\text {liq }} \times t_{d w e l l}}{6.0 \mathrm{E}^{+04}}
$$

where $\mathrm{Ag} \mathrm{Dil}_{3}$ is the mass fraction of ionic Ag in the working standards (nominal $2 \mathrm{ng} / \mathrm{g}$ to $8 \mathrm{ng} / \mathrm{g} \mathrm{Au}), q_{\text {liq }}$ is the sample flow $\left(\mathrm{g} \cdot \mathrm{min}^{-1}\right), t_{\text {dwell }}$ is the dwell time (ms).

10.1.3 Plot $I_{\mathrm{Ag} \text { ionic }}$ Vs. mass $_{\mathrm{Ag} \text { ionic }}$ for each Ag working standard, and using the regression function of Microsoft Excel, compute the slope with units of

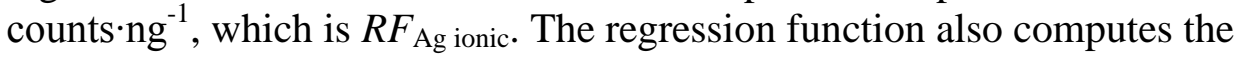
standard error of the slope which can be used in the computation of the standard uncertainty of the mass or size measurement.

10.2 Compute the mass of the 'unknown' particle, mass $_{\mathrm{NP}}(\mathrm{ng})$ 


$$
\operatorname{mass}_{N P}=\frac{\left(\left(I_{N P}-I_{\text {diss }}\right) \times \eta \mathrm{n}_{P S}\right)}{R F_{\text {Ag ionic }}}
$$

where $I_{\mathrm{NP}}$ is the intensity of the particle event, $I_{\mathrm{diss}}$ is the intensity of the dissolved background (counts, see 8.3.3.5), $\eta_{\mathrm{nPS}}$ is the transport efficiency measured via the particle size method, and $R F_{\mathrm{Ag}}$ ionic is the slope of the calibration curve formed by a plot of $I_{\mathrm{Ag} \text { ionic }} \mathrm{Vs}$. mass $_{\mathrm{Ag} \text { ionic }}$ for each $\mathrm{Ag}$ working standard (counts $\cdot \mathrm{ng}^{-1}$, see 10.1.3). Compute the diameter of the unknown' particle, $d_{\mathrm{p}}(\mathrm{nm})$

$$
d_{p}=\sqrt[3]{\frac{6 \times \operatorname{mass}_{N P}}{\pi \times \rho \times 1 \mathrm{E}^{-12}}}
$$

where mass $_{\mathrm{NP}}$ is the mass of the particle (ng, see 10.2), $\pi$ is pi and $\rho$ is the density of the particle $\left(\mathrm{g} \cdot \mathrm{cm}^{-1}\right)$.

10.3 Compute the particle size distribution

10.3.1 Compute the diameter of each particle event (sections 10.2 and 10.3).

10.3.2 Create a 'bin' column with $1 \mathrm{~nm}$ size increments spanning the observed particle size range.

10.3.2.1 Using the data analysis tool of Microsoft Excel, choose the histogram function. Choose the appropriate particle diameter and bin range data columns, specify the output range, and select chart output.

11. Calculation of the Analyte Mass Fraction $\left(\mathrm{ng} \cdot \mathrm{g}^{-1}\right)$ in the ionic fraction, $\mathrm{Con}_{\text {ionic }}$

$$
\operatorname{Con}_{\text {ionic }}=\frac{I_{\text {diss }}}{R F_{\text {ionic }} \times q_{\text {liq }} / 60 \times t_{\text {dwell }} / 1000}
$$

where $I_{\text {diss }}$ is the intensity of the dissolved or ionic fraction, corrected for instrument background (see 8.3.3.2.5.1), $R F_{\text {ionic }}$ is the slope of the calibration curve formed by a plot of $I_{\mathrm{Ag} \text { ionic }}$ Vs. mass $_{\mathrm{Ag} \text { ionic }}$ for each Ag working standard (counts'ng ${ }^{-1}$, see 10.1.3), $q_{\text {liq }}$ is the sample flow $\left(\mathrm{g} \cdot \mathrm{min}^{-1}\right)$, and $t_{\mathrm{dwell}}$ is the dwell time $(\mathrm{ms})$.

12. Computation and Uncertainty Analysis via the Kragten Spreadsheet

To gain insight into the accuracy of spICP-MS measurements and to enable comparison with established methods, the uncertainty of the measurement must be quantified. For this purpose a Kragten spreadsheet is used, because it provides a simple and practical approach to: 1 . compute a result via the equations described above, 2 . combine the uncertainties associated with each component of the measurement equation to derive an estimated expanded uncertainty, and 3. determine where the major sources of uncertainty lie so that the measurement process can be improved [12,13]. In Tables 8 through Table 11 below, example Kragten spreadsheets for the spICP-MS measurement 
of transport efficiency via the size and frequency methods, particle number concentration, and particle size are shown.

The displayed Kragten spreadsheets are color coded to draw the reader's attention to certain key areas of the spreadsheet. The first three columns highlighted in yellow describe each component of the measurement equation, list the symbol, and show typical input values. The lime green highlighted rows at the top of each spreadsheet show the estimated standard uncertainty, $u_{i}$, of each component appearing in column 2. A brief description of how standard uncertainties were evaluated for each measurement equation component appears in row 3. Uncertainty estimates are evaluated by Type A and Type B methods. Degrees of freedom, $v_{\mathrm{i}}$, for each component are shown in the fourth row, highlighted in blue. The degrees of freedom for Type A evaluations are computed as $n-$ 1 and Type $B$ evaluations are estimated to have 60 degrees of freedom. The measurement function (MF) value, or calculated result of the measurement equation, is shown in the pink row appearing under the yellow-highlighted measurement component columns. Finally, the first column of the bottom five rows of the Kragten spreadsheet show the combined standard uncertainty $\left(u_{\mathrm{c}}\right)$, the effective degrees of freedom $\left(v_{\mathrm{eff}}\right)$, the coverage factor $(k)$, the expanded uncertainty $(U)$ and the relative expanded uncertainty expressed in percent $\left(U_{\mathrm{r}}\right)$.

The combined standard uncertainty is the square root of the sum of the squares ( RSS $=\sqrt[2]{\sum_{i=1}^{n}\left(c_{i} u_{i}\right)^{2}}$ ) of the individual uncertainty components, each scaled to reflect its impact on the final result based on the measurement equation. The Kragten uses a numeric approximation to the scaled RSS to combine uncertainty components. The expanded uncertainty is calculated as $U=k u_{\mathrm{c}}$, where the value of $k$ is determined from the Student's $t$ distribution with $v_{\text {eff }}$ effective degrees of freedom. An approximately 95 $\%$ confidence interval for the measured is obtained. The effective degrees of freedom are calculated from the Welch-Satterthwaite formula (shown in [14], appendix B.3).

Adjacent to the effective degrees of freedom cell and highlighted in red font, the relative contribution of each component, calculated as its variance relative to the total variance, is shown. From this row, the reader can quickly discern which component or components most influence the overall uncertainty of the measurement. 
Table 8. Kragten Spreadsheet for Transport Efficiency via the Particle Frequency Method, $\eta_{\mathrm{nPF}}$

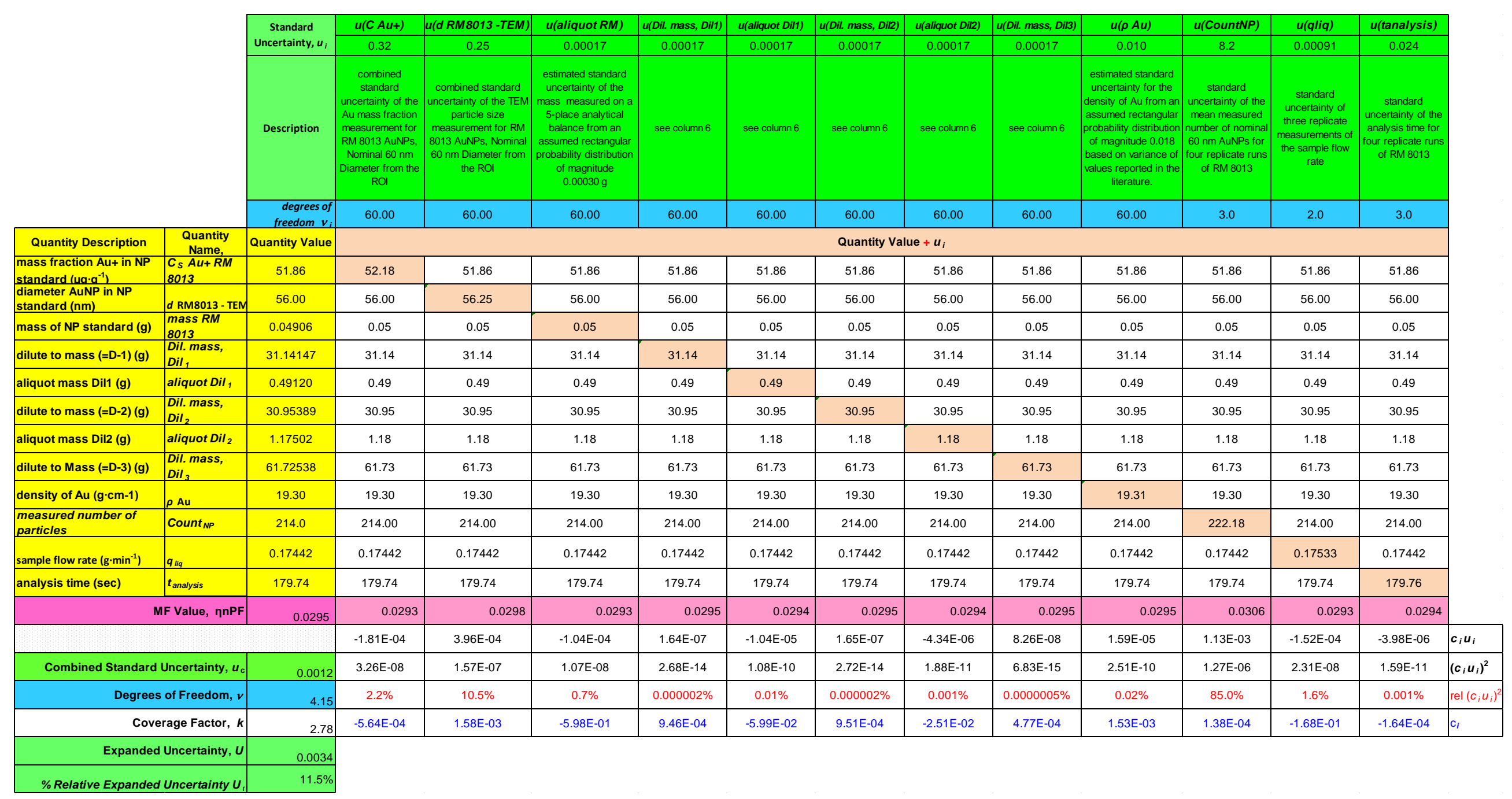


Table 9. Kragten Spreadsheet for Transport Efficiency via the Particle Size Method, $\eta_{\mathrm{nPS}}$

\begin{tabular}{|c|c|c|c|c|c|c|c|c|}
\hline & & \multirow{2}{*}{$\begin{array}{r}\text { Standard } \\
\text { Uncertainty, } u_{i}\end{array}$} & $u(R F A u$ ionic) & $u(d R M 8013-T E M)$ & $u(\rho A u)$ & $u(I N P$ AuNP) & $u($ ldiss $A u)$ & \\
\hline & & & 43183.93 & 0.25 & 0.010 & 15.5 & 0.014 & \\
\hline & & Description & $\begin{array}{l}\text { standard error of the slope } \\
\text { of the plot of } \mathrm{I}_{\mathrm{Au}} \text { ionic } \mathrm{Vs} \text {. } \\
\text { mass } \\
\text { Au ionic } \text { as computed } \\
\text { using the regression } \\
\text { function of Microsoft Excel }\end{array}$ & $\begin{array}{c}\text { combined standard } \\
\text { uncertainty of the TEM } \\
\text { particle size measurement } \\
\text { for RM } 8013 \text { AuNPs, } \\
\text { Nominal } 60 \mathrm{~nm} \text { Diameter } \\
\text { from the ROI }\end{array}$ & $\begin{array}{l}\text { estimated standard } \\
\text { uncertainty for the } \\
\text { density of Au from an } \\
\text { assumed rectangular } \\
\text { probability distribution of } \\
\text { magnitude } 0.018 \text { based } \\
\text { on variance of values } \\
\text { reported in the literature. }\end{array}$ & $\begin{array}{l}\text { standard uncertainty of } \\
\text { the mean measured } \\
\text { intensity of nominal } 60 \\
\mathrm{~nm} \text { AuNPs composed } \\
\text { of the SE of four } \\
\text { replicate runs of RM } \\
8013 \text { added in } \\
\text { quadrature to the } \\
\text { estimated SE for } \\
\text { deadtime correction }\end{array}$ & \begin{tabular}{|} 
standard \\
uncertainty of the \\
mean measured \\
intensity of the \\
dissolved $\mathrm{Au}$ \\
background for four \\
replicate runs of RM \\
8013
\end{tabular} & \\
\hline & & $\begin{array}{r}\begin{array}{r}\text { degrees of } \\
\text { freedom } v_{i}\end{array} \\
\end{array}$ & 2.00 & 60.00 & 60.00 & 19.25 & 3.00 & \\
\hline Quantity Description & \begin{tabular}{|c|} 
Quantity \\
Name, Symbol
\end{tabular} & Quantity Value & \multicolumn{5}{|c|}{ Quantity Value $+u_{i}$} & \\
\hline \multirow{2}{*}{\begin{tabular}{|l|} 
slope of the calibration \\
curve formed bv a plot of \\
diameter AuNP in NP \\
standard (nm) \\
\end{tabular}} & $R F_{A u \text { ionic }}$ & $8,709,175.69$ & $8,752,359.62$ & $8,709,175.69$ & $8,709,175.69$ & $8,709,175.69$ & $8,709,175.69$ & \\
\hline & d RM8013 - TEM & 56.00 & 56.00 & 56.25 & 56.00 & 56.00 & 56.00 & \\
\hline density of $\mathrm{Au}\left(\mathrm{g} \cdot \mathrm{cm}^{-1}\right)$ & $\rho A u$ & 19.30 & 19.30 & 19.30 & 19.31 & 19.30 & 19.30 & \\
\hline $\begin{array}{l}\text { intensity of the particle } \\
\text { events (counts) }\end{array}$ & $I_{N P} R M 8013$ & 417.57 & 417.57 & 417.57 & 417.57 & 433.06 & 417.57 & \\
\hline \begin{tabular}{|l|} 
intensity of the dissolved \\
background (counts)
\end{tabular} & $I_{\text {diss }} R M 8013$ & 0.138 & 0.14 & 0.14 & 0.14 & 0.14 & 0.15 & \\
\hline \multicolumn{2}{|r|}{ MF Value, nnPS } & 0.03703 & 0.03721 & 0.03752 & 0.03705 & 0.03570 & 0.03703 & \\
\hline & & & $1.84 \mathrm{E}-04$ & $4.98 \mathrm{E}-04$ & 1.99E-05 & $-1.33 \mathrm{E}-03$ & $1.28 \mathrm{E}-06$ & $c_{i} u_{i}$ \\
\hline \multicolumn{2}{|c|}{ Combined Standard Uncertainty, $u_{\mathrm{c}}$} & 0.00143 & 3.37E-08 & $2.48 \mathrm{E}-07$ & $3.98 \mathrm{E}-10$ & $1.76 \mathrm{E}-06$ & $1.65 \mathrm{E}-12$ & $\left(c_{i} u_{i}\right)^{2}$ \\
\hline \multicolumn{2}{|c|}{ Degrees of Freedom, $v$} & 25.67 & $1.7 \%$ & $12.2 \%$ & $0.020 \%$ & $86.2 \%$ & $0.00008 \%$ & $\operatorname{rel}\left(c_{i} u_{i}\right)^{2}$ \\
\hline \multicolumn{2}{|c|}{ Coverage Factor, $k$} & 2.06 & 4.25E-09 & 1.99E-03 & 1.92E-03 & $-8.55 E-05$ & 8.87E-05 & $c_{i}$ \\
\hline \multicolumn{2}{|c|}{ Expanded Uncertainty, $U$} & 0.0029 & & & & & & \\
\hline \multicolumn{2}{|c|}{$\%$ Relative Expanded Uncertainty $U_{\mathrm{r}}$} & $7.94 \%$ & & & & & & \\
\hline
\end{tabular}

${ }^{\mathrm{a}}$ The estimated standard error (SE) of the dead time correction was evaluated by Type $\mathrm{B}$ methods to be of magnitude $5 \%$ relative based on a DT correction of $37 \mathrm{~ns}$ for NP signals measured using $0.1 \mathrm{~ms} t_{\text {dwell }}$. 
Table 10. Kragten Spreadsheet for Determination of Particle Number Concentration of 'Unknown' AgNP Solution

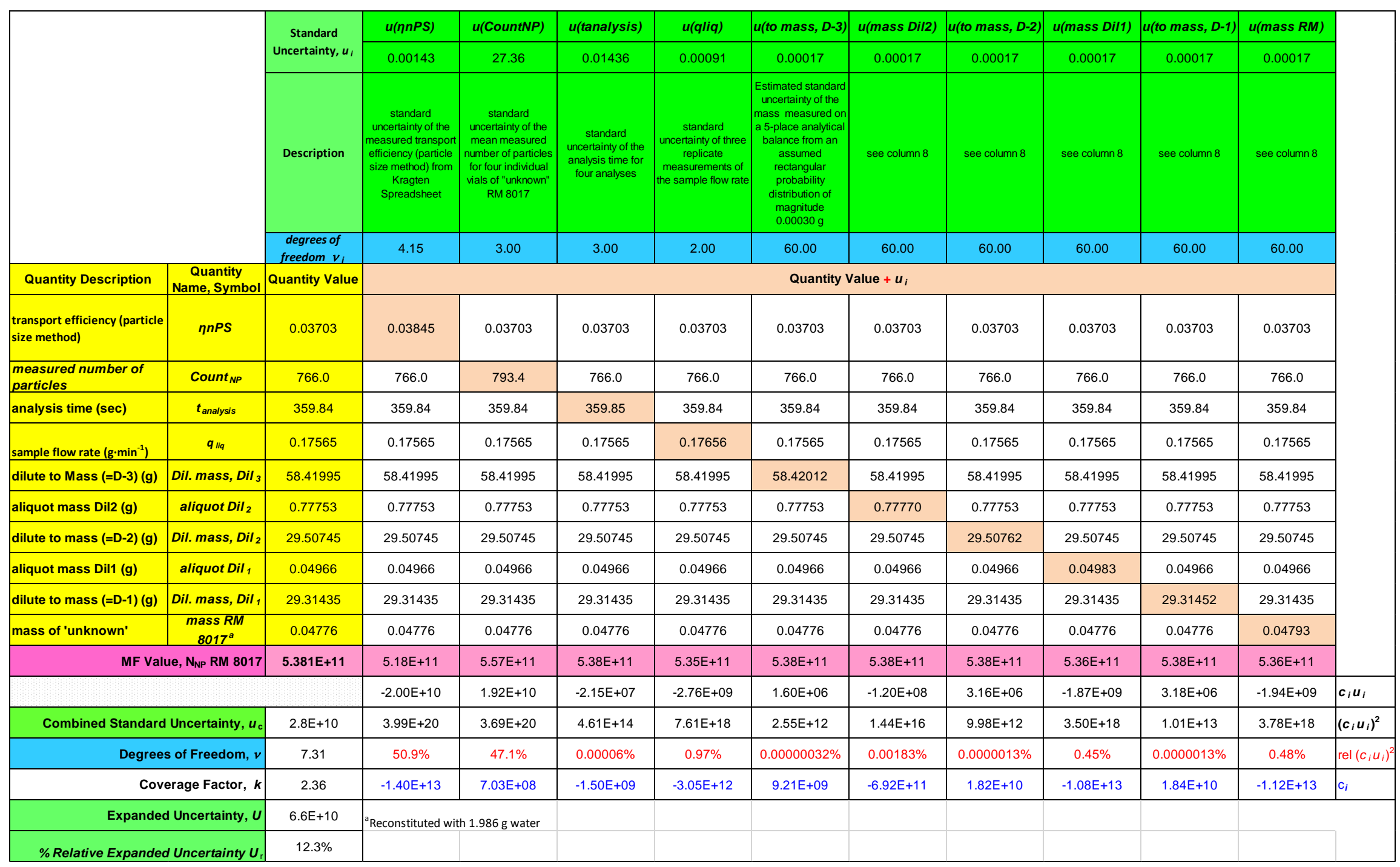


Table 11. Kragten Spreadsheet for Determination of Particle Size of 'Unknown' AgNP Solution

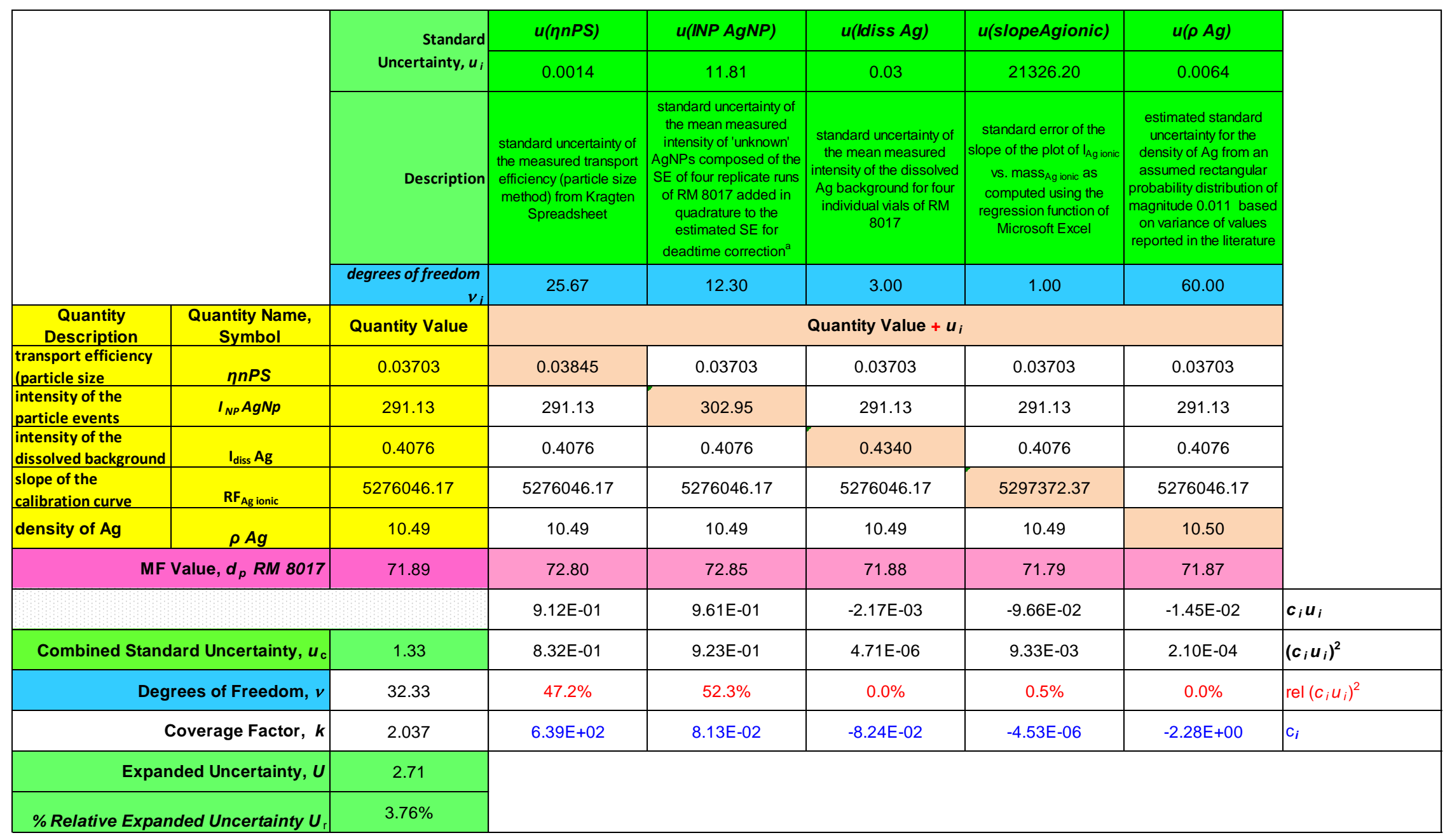

${ }^{a}$ See footnote a, Table 9. 


\section{Outcomes}

13.1 spICP-MS time resolved intensity profile and particle size distribution of RM 8017 PVP-Coated Silver Nanoparticles - Nominal Core Diameter $75 \mathrm{~nm}$

A typical time-resolved spICP-MS intensity profile and corresponding particle size distribution for a dilute suspension of a single vial of RM 8017 are shown in Figure 1. After reconstitution of the RM per instructions on the report of investigation (ROI), the suspension was diluted with water 28-million fold (see section 7.4) to a nominal particle number concentration of $1.7 \mathrm{E} 04 \mathrm{~g}^{-1}$, and measured within $0.5 \mathrm{~h}$ of dilution. The intensity of each signal pulse is proportional to the mass of analyte in a particle, and by assumption of a spherical shape, particle diameter. The number of signal pulses is proportional to the particle number concentration.

Ionic silver $\left(\mathrm{Ag}^{+}\right)$, if present in the sample suspension, can be observed in a spICP-MS time intensity profile as steady-state signal at the base of the signal pulses formed by particles. An example is shown in Figure 2, which shows spICPMS results for a dilute AgNP suspension (2.5E04 g $\mathrm{g}^{-1}, \mathrm{RM} 8017$ ) stored for $24 \mathrm{~h}$ at room temperature. The instability of the AgNP suspension under these storage conditions is evidenced by a smaller measured particle size, reduced particle number concentration, and an increased mass fraction of ionic Ag.

13.2 Repeatability, reproducibility and comparability of spICP-MS Measurements Results for the spICP-MS measurement of particle size, number concentration and ionic mass fraction in eight vials of RM 8017 that demonstrate the repeatability and reproducibility of the method are presented in Table 12 . The comparability of the spICP-MS particle size results with mean particle sizes measured by TEM, atomic force microscopy (AFM), and ultra-small angle X-ray scattering (USAXS) is illustrated in Figure 3. The comparability of spICP-MS number concentration results with a derived number concentration value for RM 8017 is shown in Figure 4.

The spICP-MS measurements were performed in separate experiments (four vials per experiment), conducted two years apart, and the presented results are calculated using both the size and frequency based measure of transport efficiency. Under repeatability conditions, a standard deviation of no greater than $\pm 1.4 \mathrm{~nm}$ was observed for the nominally $75 \mathrm{~nm}$ AgNPs. Reproducibility conditions yielded a similar standard deviation for the results using the size based measure of transport efficiency, but results calculated using the frequency based measure of transport efficiency showed a larger standard deviation $( \pm 5.1 \mathrm{~nm})$. The estimated expanded uncertainty of the size measurement (see section 12.0 and example Kragten spreadsheet in Table 11) ranged from $\pm 2.3 \mathrm{~nm}$ (3\% relative) using the particle size-based measure of transport efficiency to $\pm 8.1 \mathrm{~nm}( \pm 14 \%$ relative $)$ using the frequency-based measure of transport efficiency. The plot in Figure 3 shows that the particle size calculated using the frequency-based measure of 
transport efficiency yields results that are lower than results using the size-based measure of transport efficiency and in addition, are lower than the TEM value.

spICP-MS measured number concentration results show greater variability and poorer comparability than the spICP-MS size measurements (see Table 12 and Figure 4). Under repeatability conditions, standard deviations ranged from $\pm 1.9 \mathrm{E} 10 \mathrm{~g}^{-1}$ ( $4 \%$ relative) to $\pm 7.1 \mathrm{E} 10 \mathrm{~g}^{-1}$ (10\% relative). Standard deviations under reproducibility conditions were similar. Here again, differences in the results calculated using the two measures of transport efficiency are observed, with the frequency-based measure yielding results that deviated farthest from the reference value. The reference value for RM 8017 was computed from the TEM measured particle size, the value assigned mass of Ag in the vial, and assuming a reconstitution mass of $2.000 \mathrm{~g}$. Measured spICP-MS number concentrations using the size-based measure of transport efficiency were in good agreement with the computed number concentration reference value for RM 8017; however the measured spICP-MS number concentrations using the frequency-based measure of transport efficiency were biased high by $42 \%$ to $55 \%$. This indicates that the frequency-based measurement of transport efficiency is biased low, presumably due to loss of AuNPs. Analysis of the total Au mass fraction via acid digestion of a spICP-MS suspension diluted to $1.6 \mathrm{E} 4 \mathrm{~g}^{-1}$ that was used to measure transport efficiency did show that the measured Au mass fraction was significantly lower than expected, indicating loss of $\mathrm{Au}$ to the container walls. A more rigorous study of the stability of the very dilute suspension required for spICP-MS measurements with respect to particle number concentration, is needed. It is worthy to note that the size-based measurement of transport efficiency is more robust, as it is unaffected by particle loss to the container walls.

The Ag ion content measured in each vial of RM 8017 and expressed as the fraction of total Ag is listed in the last column of Table 12. It should be noted that RM 8017 is not an ideal sample for measurement of the component Ag ion content. Since RM 8017 is a freeze-dried material that is designed to be reconstituted and used within as short time frame, a reference value for the Ag ion content of RM 8017 has not been established. Furthermore due to the high AgNP concentration and subsequent large dilution (nominally 28 million-fold) required to achieve the optimum number concentration for single particle analysis, the ionic Ag content, if any, has been so diluted that the measured signals are at the method detection limit and subject to high uncertainty. This is evident in the observed variability of the results. Measurement of the ionic fraction of a less concentrated solution with respect to particle number, would presumably yield more consistent results. 
Table 12. Repeatability and Reproducibility of spICP-MS Measurements of Particle Size, Number Concentration and Ionic Mass Fraction of RM 8017 PVP-Coated Silver Nanoparticles - Nominal Diameter 75 nm

\begin{tabular}{|c|c|c|c|c|c|c|c|c|}
\hline \multirow[b]{2}{*}{$\begin{array}{c}\text { Analysis } \\
\text { Date }\end{array}$} & \multirow[b]{2}{*}{ Vial\# } & \multirow{2}{*}{$\begin{array}{l}\text { Analysis } \\
\text { time, } t_{\text {analysis }} \\
\text { (s) }\end{array}$} & \multirow{2}{*}{$\begin{array}{c}\text { Number of } \\
\text { counted } \\
\text { particles, } \\
\text { Count }_{N P}\end{array}$} & \multicolumn{2}{|c|}{ Particle Diameter, $d_{P}(\mathrm{~nm})$} & \multicolumn{2}{|c|}{ Particle Concentration, $N_{P}\left(\mathrm{~g}^{-1}\right)$} & \multirow{2}{*}{$\begin{array}{c}\text { Ag Ionic } \\
\text { Fraction, } \\
\boldsymbol{A g}_{\text {ionic }} \\
(\%)^{\mathrm{a}}\end{array}$} \\
\hline & & & & TE by size & $\begin{array}{c}\text { TE by } \\
\text { frequency }\end{array}$ & TE by size & $\begin{array}{c}\text { TE by } \\
\text { frequency }\end{array}$ & \\
\hline \multirow{7}{*}{2013} & $1^{b}$ & 99.79 & 297 & 68.96 & 58.29 & $4.67 \mathrm{E}+11$ & $7.73 \mathrm{E}+11$ & 5.6 \\
\hline & $2^{c}$ & 179.70 & 275 & 68.24 & 58.19 & $4.39 \mathrm{E}+11$ & $7.08 \mathrm{E}+11$ & 7.0 \\
\hline & $3^{c}$ & 179.71 & 362 & 69.90 & 58.75 & $5.01 \mathrm{E}+11$ & $8.08 \mathrm{E}+11$ & 2.9 \\
\hline & $4^{\mathrm{c}}$ & 179.75 & 256 & 70.32 & 59.97 & $4.02 \mathrm{E}+11$ & $6.49 \mathrm{E}+11$ & 1.2 \\
\hline & & & Mean & 69.1 & 58.8 & $4.52 \mathrm{E}+11$ & $7.30 \mathrm{E}+11$ & 4.2 \\
\hline & & & SD & 0.9 & 0.8 & $0.42 \mathrm{E}+11$ & $0.71 \mathrm{E}+11$ & 2.6 \\
\hline & & & $\boldsymbol{U}$ & 2.3 & 8.1 & $0.65 \mathrm{E}+11$ & $2.3 \mathrm{E}+11$ & \\
\hline & & & & & & & & \\
\hline \multirow{9}{*}{2015} & $5^{\mathrm{d}}$ & 359.84 & 766 & 71.89 & 66.68 & $5.34 \mathrm{E}+11$ & $6.69 \mathrm{E}+11$ & 3.45 \\
\hline & $6^{\mathrm{d}}$ & 359.78 & 675 & 71.52 & 66.35 & $4.87 \mathrm{E}+11$ & $6.11 \mathrm{E}+11$ & 3.78 \\
\hline & $7^{\mathrm{d}}$ & 359.84 & 789 & 72.05 & 66.83 & $5.55 \mathrm{E}+11$ & $6.95 \mathrm{E}+11$ & 4.95 \\
\hline & $8^{d}$ & 359.83 & 846 & 69.00 & 64.00 & $5.77 \mathrm{E}+11$ & $7.23 \mathrm{E}+11$ & 5.01 \\
\hline & & & Mean & 71.1 & 65.97 & $5.38 \mathrm{E}+11$ & $6.70 \mathrm{E}+11$ & 4.30 \\
\hline & & & SD & 1.4 & 1.3 & 0.19E+11 & $0.24 \mathrm{E}+11$ & 0.80 \\
\hline & & & $\boldsymbol{U}$ & 2.7 & 2.7 & $0.66 \mathrm{E}+11$ & $1.1 \mathrm{E}+11$ & \\
\hline & \multicolumn{3}{|c|}{ Reproducibility SD } & 1.4 & 5.1 & $0.61 \mathrm{E}+11$ & $0.42 \mathrm{E}+11$ & \\
\hline & \multicolumn{3}{|c|}{ Reference Value } & $74.6 \pm$ & $8^{e} \mathrm{~nm}$ & \multicolumn{2}{|c|}{$4.75 \mathrm{E}+11 \pm 0.68 \mathrm{E}+11^{\mathrm{f}}$} & \\
\hline
\end{tabular}

${ }^{a}$ Expressed as the fraction of total $\mathrm{Ag}$

${ }^{\mathrm{b}}$ Nominal analysis $N_{P}, 2.4 \mathrm{E} 04 \mathrm{~g}^{-1}$, ${ }^{\mathrm{c}}$ Nominal analysis $N_{P}, 1.4 \mathrm{E} 04 \mathrm{~g}^{-1}$, ${ }^{\mathrm{d}}$ Nominal analysis $N_{P}, 1.7 \mathrm{E} 04 \mathrm{~g}^{-1}$

${ }^{\mathrm{e}}$ Value Assigned TEM size $\pm U$ for RM 8017

${ }^{\mathrm{f}}$ Derived $N_{\mathrm{P}} \pm U$ for RM 8017 calculated based on TEM size, mass Ag in vial, and reconstitution mass of $2.000 \mathrm{~g}$. Assumes all Ag is particulate. $U$ calculated via Kragten spreadsheet. 
14 Abbreviations

$\begin{array}{ll}\text { AFM } & \text { Atomic Force Microscopy } \\ \text { AgNPs } & \text { Silver nanoparticles } \\ \text { AuNPs } & \text { Gold nanoparticles } \\ \text { DI } & \text { Deionized } \\ \text { Nano-EHS } & \text { Environmental, Health and Safety as it pertains to nanomaterials } \\ \text { NNI } & \text { National Nanotechnology Initiative } \\ \text { spICP-MS } & \text { Single Particle Inductively Coupled Plasma Mass Spectrometry } \\ \text { TEM } & \text { Transmission Electron Microscopy (TEM) } \\ \text { USAXS } & \text { Ultra Small Angle X-ray Scattering }\end{array}$

15 Acknowledgements

We would like to thank Debra Kaiser for her direction and vision in the coordination of the implementation of the National Nanotechnology Initiative Nano-EHS research strategy at the National Institute of Standards and Technology from which the idea for the development of this series of protocols originated.

16 References

1. National Nanotechnology Initiative Environmental, Health, and Safety Research Strategy, 2011, http://www.nano.gov/node/1157.

2. Degueldre C. and Favarger P.Y., Colloid analysis by single particle inductively coupled plasmamass spectroscopy: a feasibility study. Colloids Surf., A, 2003, 217, 137-142.

3. Olesik J. and Gray P. Considerations for measurement of individual nanoparticles or microparticles by ICP-MS: determination of the number of particles and the analyte mass in each particle. J. Anal. At. Spectrom., 2012, 27, 1143 - 1155.

4. Laborda F., Jimenez-Lamana J., Bolea E., and Castillo J. Selective identification, characterization and determination of dissolved silver(I) and silver nanoparticles based on single particle detection by inductively coupled plasma mass spectrometry. J. Anal. At. Spectrom., 2011, 26, 1362-1371.

5. Gschwind S., Hagendorfer H., Frick D., and Günther D. Mass Quantification of Nanoparticles by Single Droplet Calibration Using Inductively Coupled Plasma Mass Spectrometry. Anal. Chem., 2013, 85, 5875-5883.

6. $\quad$ Pace H., Rogers N., Jarolimek C., Coleman V., Higgins C., and Ranville J. Determining Transport Efficiency for the Purpose of Counting and Sizing Nanoparticles via Single Particle Inductively Coupled Plasma Mass Spectrometry. Anal. Chem., 2011, 83, 93619369.

7. Liu J., Murphy K., MacCuspie R., and Winchester M. Capabilities of Single Particle Inductively Coupled Plasma Mass Spectrometry for the Size Measurement of Nanoparticles: A Case Study on Gold Nanoparticles. Anal. Chem., 2014, 86, 3405-3414.

8. ISO/TS 13278:2011, Nanotechnologies - Determination of elemental impurities in samples of carbon nanotubes using inductively coupled plasma mass

9. ISO/TS 27687:2008, 4.1, Nanotechnologies - Terminology and definitions for nanoobjects: Nanoparticle, nanofibre and nanoplate 
10. Laborda F., Jimenez-Lamana J., Bolea E., Castillo J. Critical considerations for the determination of nanoparticle number concentrations, size and number size distributions by single particle ICP-MS. J. Anal. At. Spectrom., 2013, 28, 1220-1232.

11. Tuoriniemi J., Cornelis, Hassellov M. Improving the accuracy of single particle ICPMS for measurement of size distributions and number concentrations of nanoparticles by determining analyte partitioning during nebulisation. J. Anal. At. Spectrom., 2014, 29, 743 -752 .

12. Kragten, J. Calculating standard deviations and confidence intervals with a universally applicable spreadsheet technique. Analyst, 1994, 119, 2161-2165.

13. Vetter, T.W. Quantifying measurement uncertainty in analytical chemistry - a simplified approach. Proceedings of the Measurement Science Conference, Paper V-B, January 2001, Anaheim CA; also see http://www.p2pays.org/ref/18/17628.pdf.

14. B. N. Taylor and C. E. Kuyatt. Guidelines for Evaluating and Expressing the Uncertainty of NIST Measurement Results. National Institute of Standards and Technology, Gaithersburg, MD, 1994. URL http://physics.

nist.gov/Pubs/guidelines/TN1297/tn1297s.pdf. NIST Technical Note 1297. 

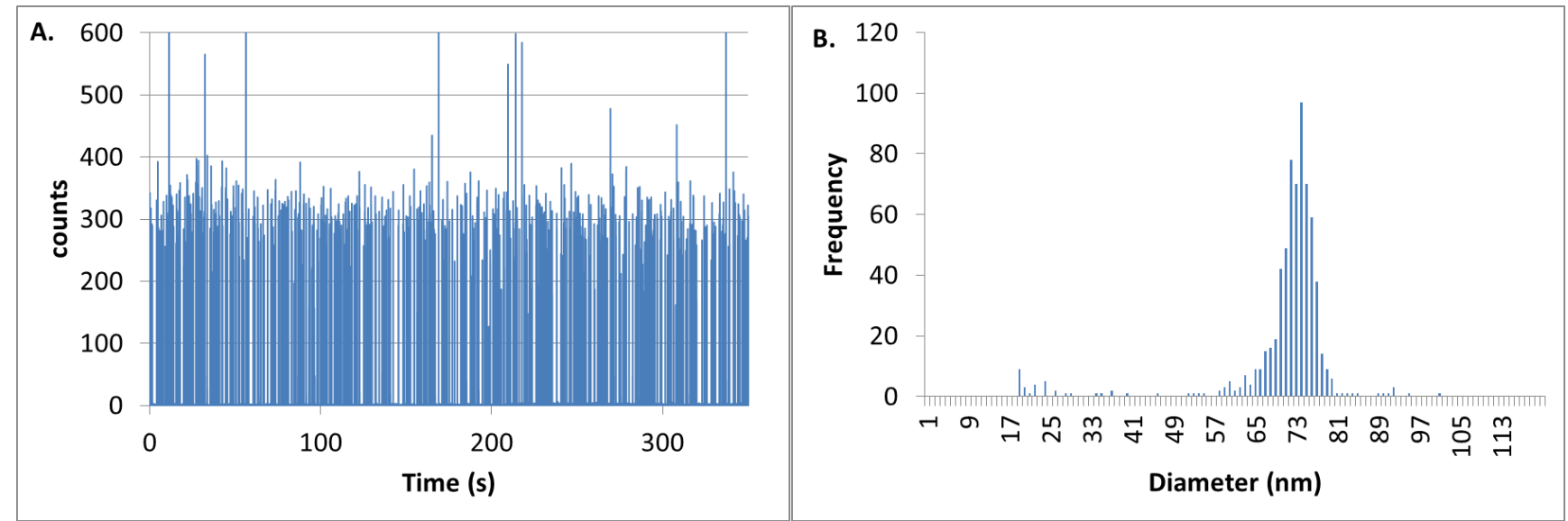

Figure 1. Time-resolved intensity profile (A) and corresponding particle size distribution (B) for a spICP-MS suspension $\left(1.7 \mathrm{E} 04 \cdot \mathrm{g}^{-1}\right)$ of a single vial of RM 8017, nominally $75 \mathrm{~nm}$ AgNPS measured within $0.5 \mathrm{~h}$ of dilution.
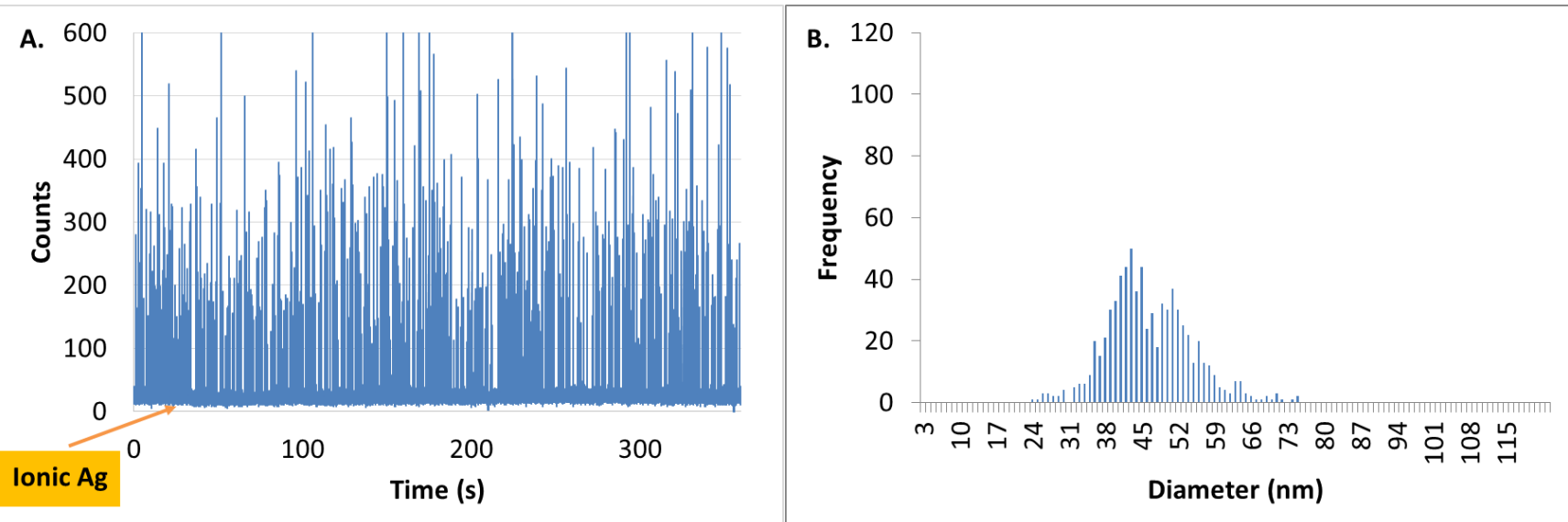

Figure 2. Time-resolved intensity profile (A) and corresponding particle size distribution (B) for a spICP-MS suspension (2.5E07 $\left.\mathrm{g}^{-1}\right)$ of AgNPS (RM 8017) stored at room temperature for $24 \mathrm{~h}$. The instability of the dilute suspension under these conditions is evidenced by a decrease in measured particle size and number concentration, and an increase in the mass fraction of ionic Ag. 


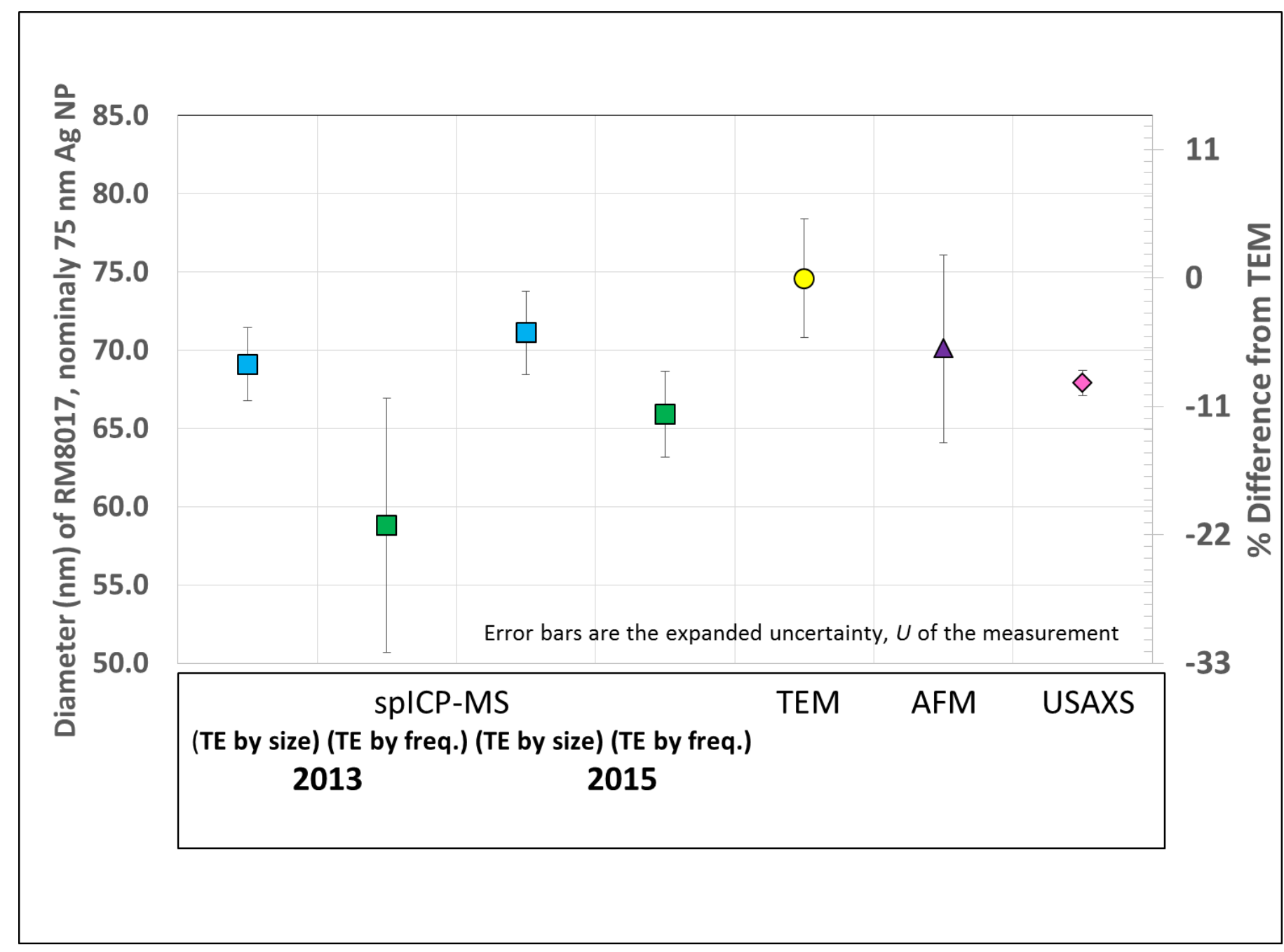

Figure 3. Comparability of the spICP-MS particle size results for RM 8017 calculated using both the size-based and frequency-based measure of transport efficiency with mean particle sizes measured by TEM, AFM, and USAXS 


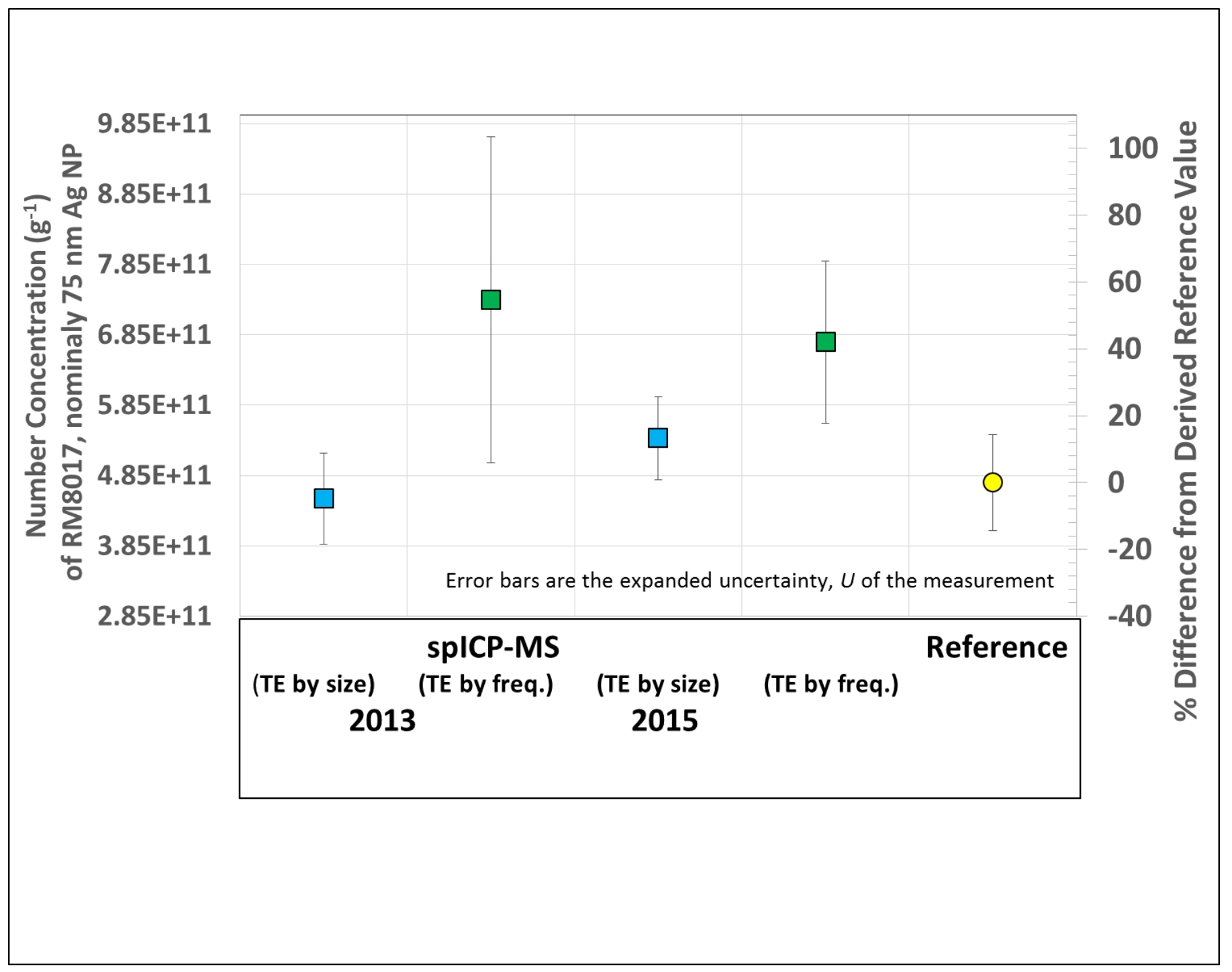

Figure 4. Comparability of the spICP-MS number concentration results for RM 8017 calculated using both the size-based and frequency-based measure of transport efficiency with the derived reference value based on TEM - measured AgNP size, mass Ag in vial, and reconstitution mass of $2.000 \mathrm{~g}$. 